\title{
Parallelogram decompositions and generic surfaces in $\mathcal{H}^{\text {hyp }}(4)$
}

\author{
DUC-MANH NGUYEN
}

The space $\mathcal{H}^{\text {hyp }}(4)$ is the moduli space of pairs $(M, \omega)$, where $M$ is a hyperelliptic Riemann surface of genus 3 and $\omega$ is a holomorphic 1 -form having only one zero. In this paper, we first show that every surface in $\mathcal{H}^{\text {hyp }}(4)$ admits a decomposition into parallelograms and simple cylinders following a unique model. We then show that if this decomposition satisfies some irrational condition, then the $\mathrm{GL}^{+}(2, \mathbb{R})$-orbit of the surface is dense in $\mathcal{H}^{\text {hyp }}(4)$; such surfaces are called generic. Using this criterion, we prove that there are generic surfaces in $\mathcal{H}^{\text {hyp }}(4)$ with coordinates in any quadratic field, and there are Thurston-Veech surfaces with trace field of degree three over $\mathbb{Q}$ which are generic.

51H25; 37B05

\section{Introduction}

Translation surfaces are flat surfaces with conical singularities and trivial linear holonomy, that is, the holonomy of any closed curve is a translation in $\mathbb{R}^{2}$. The space of translation surfaces together with an oriented parallel line field is identified with the space of holomorphic 1-forms on Riemann surfaces, which is stratified by the orders of the zeros of the 1 -form. Fixing $g \geqslant 2$, if $k_{1}, \ldots, k_{n}$ are positive integers such that $k_{1}+\cdots+k_{n}=2 g-2$, we denote by $\mathcal{H}\left(k_{1}, \ldots, k_{n}\right)$ the moduli space of holomorphic 1 -forms on Riemann surfaces of genus $g$ which have exactly $n$ zeros with orders $\left(k_{1}, \ldots, k_{n}\right)$. By a result of Kontsevich and Zorich [9], we know that $\mathcal{H}\left(k_{1}, \ldots, k_{n}\right)$ has at most 3 connected components. We denote by $\mathcal{H}_{1}\left(k_{1}, \ldots, k_{n}\right)$ the subset of $\mathcal{H}\left(k_{1}, \ldots, k_{n}\right)$ consisting of surfaces of unit area. On $\mathcal{H}\left(k_{1}, \ldots, k_{n}\right)$, one has a volume form which is the pullback of the Lebesgue measure in the local charts defined by the period mappings. This volume form induces naturally a volume form on $\mathcal{H}_{1}\left(k_{1}, \ldots, k_{n}\right)$. By results of Masur [10] and Veech [15] (see also Eskin and Okounkov [4]), we know that the volume of $\mathcal{H}_{1}\left(k_{1}, \ldots, k_{n}\right)$ is finite.

There is an action of $\mathrm{GL}^{+}(2, \mathbb{R})$ on the space $\mathcal{H}\left(k_{1}, \ldots, k_{n}\right)$. The action of the subgroup $\operatorname{SL}(2, \mathbb{R})$ leaves invariant the Lebesgue measure and preserves the set $\mathcal{H}_{1}\left(k_{1}, \ldots, k_{n}\right)$. It is now a classical fact, also due to Masur and Veech, that the 
$\operatorname{SL}(2, \mathbb{R})$ action is ergodic in each component of $\mathcal{H}_{1}\left(k_{1}, \ldots, k_{n}\right)$. A surface of unit area whose $\operatorname{SL}(2, \mathbb{R})$-orbit is dense in a component of $\mathcal{H}_{1}\left(k_{1}, \ldots, k_{n}\right)$ (or equivalently, whose $\mathrm{GL}^{+}(2, \mathbb{R})$-orbit is dense in a component of $\left.\mathcal{H}\left(k_{1}, \ldots, k_{n}\right)\right)$ is called generic.

Knowledge about the $\operatorname{SL}(2, \mathbb{R})$-orbit closures is useful since it is strongly related to asymptotic behaviors of the linear flow on individual surfaces (see Zorich [16, Section 3.6]). Despite the fact that almost all surfaces are generic, the problem of determining whether the orbit of a particular surface is dense in its component is wide open. We only have a complete classification (due to McMullen [12] and Calta [1]) for the case of genus 2 , where we have two strata, $\mathcal{H}(2)$ and $\mathcal{H}(1,1)$, each of which has a single connected component. Recall that the Veech group of a translation surface is the stabilizer subgroup for the action of $\operatorname{SL}(2, \mathbb{R})$. It is a well-known fact (see Masur and Tabachnikov [11], Zorich [16] and McMullen [12]) that the $\operatorname{SL}(2, \mathbb{R})$-orbit of a surface is a closed subset in its stratum if and only if its Veech group is a lattice of $\operatorname{SL}(2, \mathbb{R})$. It turns out from the work of McMullen that, for translation surfaces of genus two, if the Veech group contains a hyperbolic element, then the $\operatorname{SL}(2, \mathbb{R})$-orbit cannot be dense in the corresponding stratum.

More recently, Hubert, Lanneau and Möller [6; 5] give some results on generic surfaces in the hyperelliptic locus $\mathcal{L}$ in $\mathcal{H}^{\text {odd }}(2,2)$, which is one of the two components of $\mathcal{H}(2,2)$. They show that, in contrast with the case of genus 2 , there are generic surfaces in $\mathcal{L}$, that is, the $\operatorname{SL}(2, \mathbb{R})$-orbit is dense in $\mathcal{L}$, whose Veech group contains hyperbolic elements. Note that $\mathcal{L}$ is a closed, $\operatorname{SL}(2, \mathbb{R})$-invariant subset of $\mathcal{H}^{\text {odd }}(2,2)$, therefore, the closure of any $\operatorname{SL}(2, \mathbb{R})$-orbit in $\mathcal{L}$ cannot exceed $\mathcal{L}$. The ThurstonVeech construction $[13 ; 14]$ provides us with translation surfaces which are stabilized by some hyperbolic elements in $\operatorname{SL}(2, \mathbb{R})$, these hyperbolic elements arise as products of parabolic ones. Hubert, Lanneau and Möller also show that there are surfaces in $\mathcal{L}$ obtained from the Thurston-Veech construction whose $\operatorname{SL}(2, \mathbb{R})$-orbit is dense in $\mathcal{L}$.

The stratum $\mathcal{H}(4)$ is the space of holomorphic 1 -form on Riemann surfaces of genus 3 which have only one zero (the order is necessarily 4 ). We have $\operatorname{dim}_{\mathbb{C}} \mathcal{H}(4)=6$, and $\mathcal{H}(4)$ has two connected components $\mathcal{H}^{\text {hyp }}(4)$ and $\mathcal{H}^{\text {odd }}(4)$ (see Kontsevich and Zorich [9]). In this paper, we will be focusing on the connected component $\mathcal{H}^{\text {hyp }}(4)$ which consists of holomorphic 1-forms defined on hyperelliptic Riemann surfaces. Note that the unique zero of the 1-form is necessarily a Weierstrass point. Equivalently, we can consider $\mathcal{H}^{\text {hyp }}(4)$ as the space of translation surfaces of genus 3 having only one singularity, such that there exists an isometric involution which has exactly 8 fixed points, and acts by $-\mathrm{Id}$ on the homology.

Before stating the main results of this paper, let us recall some basic definitions. On a translation surface, a saddle connection is a geodesic segment whose endpoints are 
singularities of the surface, which may coincide. For surfaces in $\mathcal{H}^{\text {hyp }}(4)$, a saddle connection is then a geodesic loop joining the unique singularity to itself. If $\gamma$ is a saddle connection, we denote its length by $|\gamma|$. We can also associate to $\gamma$ together with a choice of orientation a vector $V(\gamma) \in \mathbb{R}^{2}$ which is the integral of the holomorphic 1 -form defining the flat metric along $\gamma$. In fact, the integral gives us a complex number that we regard as a vector in $\mathbb{R}^{2}$ by the standard identification $\mathbb{C}=\mathbb{R} \oplus i \mathbb{R}$.

Given a translation surface $\Sigma$, a cylinder in $\Sigma$ is an open subset which is isometric to the quotient $\mathbb{R} \times] 0 ; h[/ \mathbb{Z}$, where $\mathbb{Z}$ is the cyclic group generated by $(x, y) \mapsto(x+\ell, y)$, and maximal with respect to this property. We will call $h$ the height, and $\ell$ the width of $C$, and define the modulus of $C$ to be the ratio $h / \ell$. Note that none of the parameters $h, \ell, m$ are invariant under $\operatorname{SL}(2, \mathbb{R})$. By definition, we have a map from $\mathbb{R} \times] 0 ; h[$ to $\Sigma$, which is locally isometric, with image $C$. This map can be extended by continuity to a map from $\mathbb{R} \times[0 ; h]$ to $\Sigma$. We call the images of $\mathbb{R} \times\{0\}$ and $\mathbb{R} \times\{h\}$ under this map the boundary components of $C$. Each boundary component of $C$ is a concatenation of saddle connections, and freely homotopic to the simple closed geodesics in $C$. Observe that the two boundary components of $C$ are, in general, not disjoint subsets of $\Sigma$ - they can even coincide. We call $C$ a simple cylinder when each of its boundary components consists of only one saddle connection.

A direction $\theta$ in $\mathbb{S}^{1}$ is said to be completely periodic if $\Sigma$ is the union of the closures of the cylinders in this direction, in other words, any trajectory of the flow in this direction, is either a closed geodesic or a saddle connection.

Theorem 1.1 On every surface in $\mathcal{H}^{\text {hyp }}(4)$, there always exist four pairs of homologous saddle connections $\delta_{i}^{ \pm}, i=1, \ldots, 4$, such that

- $\delta_{1}^{ \pm}$bound a simple cylinder,

- $\delta_{i}^{ \pm}$and $\delta_{i+1}^{ \pm}$bound a topological disk which is isometric to a parallelogram in $\mathbb{R}^{2}$ for $i=1,2,3$,

- $\delta_{4}^{ \pm}$bound a simple cylinder.

The configuration of $\delta_{1}^{ \pm}, \ldots, \delta_{4}^{ \pm}$is shown in Figure 1 .

Let $\Sigma_{0}$ be a surface in $\mathcal{H}_{1}^{\text {hyp }}(4)$, and $\delta_{i}^{ \pm}, i=1, \ldots, 4$, be as in Theorem 1.1. Cutting $\Sigma$ along $\delta_{3}^{ \pm}$, we get two connected components whose boundary consists of two geodesic segments. Gluing those geodesic segments together, we then get a flat torus, which will be denoted by $\Sigma^{\prime}$, and a surface in $\mathcal{H}(2)$. On the torus $\Sigma^{\prime}$, we denote the geodesic segment corresponding to $\delta_{3}^{ \pm}$by $\delta_{3}$. As a subsurface of $\Sigma$, the torus $\Sigma^{\prime}$ inherits a parallel line field, therefore we can view it as a pair $(M, \omega)$, where $M$ is a Riemann 


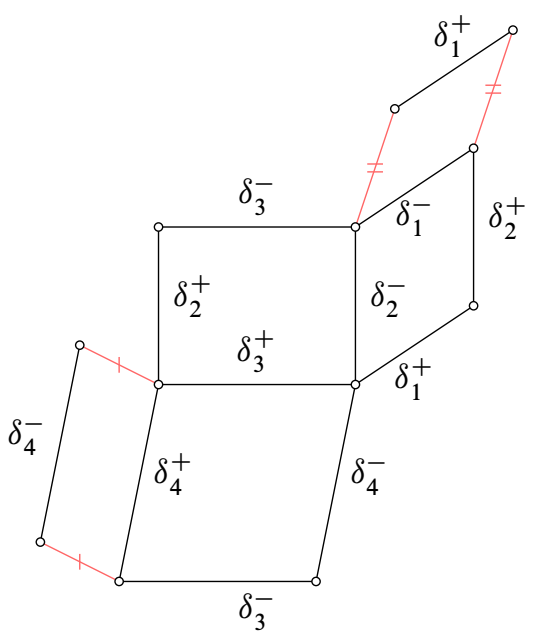

Figure 1: Decomposition into parallelograms and simple cylinders

surface of genus one, and $\omega$ is a nonzero holomorphic 1-form on $M$. Equivalently, we can identify $\Sigma^{\prime}$ with the quotient $\mathbb{R}^{2} / \Lambda$, where $\Lambda$ is a lattice in $\mathbb{R}^{2}$, which is the image of the map $H_{1}(M, \mathbb{Z}) \longrightarrow \mathbb{C} \simeq \mathbb{R}^{2}: c \mapsto \int_{c} \omega$. A vector in $\mathbb{R}^{2}$ is said to be generic with respect to $\Lambda$ if it is not collinear with any vector in $\Lambda$. We have

Theorem 1.2 Suppose that $\delta_{1}^{ \pm}$and $\delta_{3}^{ \pm}$are parallel, that is $V\left(\delta_{1}^{ \pm}\right)$and $V\left(\delta_{3}^{ \pm}\right)$are colinear, and $V\left(\delta_{3}\right)=V\left(\delta_{3}^{ \pm}\right)$is generic with respect to $\Lambda$. Then $\operatorname{SL}(2, \mathbb{R}) \cdot \Sigma_{0}$ is dense in $\mathcal{H}_{1}^{\text {hyp }}(4)$.

Using this result, one can show that:

Corollary 1.3 Let $\Sigma_{0}$ be a surface in $\mathcal{H}_{1}^{\text {hyp }}(4)$. Suppose that the horizontal direction is completely periodic for $\Sigma_{0}$, and that $\Sigma_{0}$ is decomposed into three horizontal cylinders whose moduli are independent over $\mathbb{Q}$. Then $\operatorname{SL}(2, \mathbb{R}) \cdot \Sigma_{0}$ is dense in $\mathcal{H}_{1}^{\text {hyp }}(4)$.

Remark Since the condition that $V\left(\delta_{1}^{ \pm}\right)$and $V\left(\delta_{3}^{ \pm}\right)$are colinear are not generic, the set of surfaces in $\mathcal{H}_{1}^{\text {hyp }}(4)$ satisfying the hypothesis of Theorem 1.2 has measure zero.

The proof of Theorem 1.1 relies on the action of the hyperelliptic involution on the surfaces in $\mathcal{H}^{\text {hyp }}(4)$. The key ingredient of the proof is Lemma 2.1, which says that, on a translation surface of genus one or two, any saddle connection invariant under the distinguished involution of the surface is contained in a simple cylinder.

To prove Theorem 1.2, we will show that the orbit closure contains all the surfaces admitting a splitting as in Theorem 1.1 with $\delta_{2}^{ \pm}$parallel to $\delta_{3}^{ \pm}$. Consequently, the orbit 
closure contains all the Veech surfaces, and in particular all the square-tiled surfaces. Since the set of square-tiled surfaces is dense in $\mathcal{H}_{1}^{\text {hyp }}$ (4) (see for example Douady and Hubbard [3] or Kontsevich and Zorich [9]), we deduce that the orbit closure is the whole component. The proof of Theorem 1.2 uses a theorem of Ratner on action of unipotent subgroups on homogeneous spaces.

To prove Corollary 1.3, we prove that one can find in the $\operatorname{SL}(2, \mathbb{R})$-orbit closure of $\Sigma_{0}$ a surface which satisfies the condition of Theorem 1.2. Using Theorem 1.2, and Corollary 1.3, we can find numerous interesting examples of generic surfaces in $\mathcal{H}^{\text {hyp }}(4)$, in particular, surfaces with coordinates in any quadratic field over $\mathbb{Q}$. Therefore, we have an affirmative answer to a question of Hubert, Lanneau and Möller [7] (see Section 9). We will also construct explicitly some Thurston-Veech surfaces with trace field of degree three over $\mathbb{Q}$ which satisfy the hypothesis of Corollary 1.3.

Acknowledgements This work was started during the author's stay at Max-PlanckInstitut für Mathematik in Bonn, the author is grateful to the Institute for its hospitality. The author warmly thanks Erwan Lanneau and Martin Möller for the advices and stimulating discussions.

This work is dedicated to Professor Nguyễn Duy Tiến on the occasion of his 70th birthday, with gratefulness and affection.

\section{Simple cylinder invariant under the involution}

\subsection{Translation surfaces of genus one}

Translation surfaces of genus one are simply flat tori. We denote by $\mathcal{H}(0)$ (resp. $\mathcal{H}(0,0))$ the space of triples $(M, \omega, p)$ (resp. quadruplet $\left.\left(M, \omega, p_{1}, p_{2}\right)\right)$, where $M$ is a Riemann surface of genus one (an elliptic curve), $\omega$ is a nonzero holomorphic 1 -form on $M$, and $p$ (resp. $p_{1}$ and $p_{2}$ ) is a marked point (resp. are marked points) of $M$. In both cases, we will call the lattice in $\mathbb{R}^{2}$ obtained by integrating $\omega$ along elements of $H_{1}(M, \mathbb{Z})$ the associated lattice of the considered translation surface. If $\Sigma$ is an element of $\mathcal{H}(0)$ or $\mathcal{H}(0,0)$, we denote by $\Lambda(\Sigma)$ the lattice associated to $\Sigma$.

Note that the holomorphic 1-form determines a flat metric structure together with a choice of vertical direction at every point of the surface. For each surface in $\mathcal{H}(0)$, and $\mathcal{H}(0,0)$, we have a distinguished isometric involution which acts like -Id on the homology of the surface, and either fixes the unique marked point in the case of $\mathcal{H}(0)$, or exchanges the two marked points in the case of $\mathcal{H}(0,0)$. As usual, we will call a geodesic segment joining marked points a saddle connection. In the case of $\mathcal{H}(0)$, a saddle connection is just a simple closed geodesic passing through the marked point. 


\subsection{Saddle connection preserved by the involution}

Let us first prove the following lemma, which is the key ingredient for the proof of Theorem 1.1:

Lemma 2.1 Let $\gamma$ be a saddle connection on a translation surface $\Sigma$ which belongs to one of the following strata $\mathcal{H}(0), \mathcal{H}(0,0), \mathcal{H}(2), \mathcal{H}(1,1)$. Suppose that $\gamma$ is invariant under the distinguished involution in the cases $\mathcal{H}(0)$ and $\mathcal{H}(0,0)$, or under the hyperelliptic involution in the cases $\mathcal{H}(2)$ and $\mathcal{H}(1,1)$. Then there exists a pair of saddle connections $\left(\eta^{+}, \eta^{-}\right)$which bound a simple cylinder $C$ containing $\gamma$. In the case $\mathcal{H}(0)$, we have $\eta^{+} \equiv \eta^{-}$, in all other cases $\eta^{+}$and $\eta^{-}$are distinct saddle connections.

Proof We will prove this lemma case by case.

Case $\mathcal{H}(0)$ In this case $\gamma$ is a simple closed geodesic passing through the marked point. Let $\eta$ be any simple closed geodesic which meets $\gamma$ only at the marked point. Then we can take $\eta^{+}=\eta^{-}=\eta$.

Case $\mathcal{H}(0,0)$ In this case $\gamma$ is a geodesic segment joining two marked points $p_{1}, p_{2}$ of $\Sigma$. Let $\tau$ denote the distinguished involution of $\Sigma$. If $\eta^{+}$is a simple closed geodesic which meets $\gamma$ only at $p_{1}$, then $\eta^{-}=\tau\left(\eta^{+}\right)$is also a simple closed geodesic freely homotopic to $\eta^{+}$, and $\eta^{+} \cup \eta^{-}$is boundary of a simple cylinder containing $\gamma$ which is invariant under $\tau$.

Using the action of $\operatorname{SL}(2, \mathbb{R})$, we can assume that $\gamma$ is horizontal. To see that there always exists a simple closed geodesic which meets $\gamma$ only at $p_{1}$, we consider the vertical flow $\Psi_{t}, t \in \mathbb{R}$, on $\Sigma$. There exists a minimal value $t_{0}>0$ such that $\Psi_{t_{0}}(\gamma)$ intersects $\gamma$. Observe that $\Psi_{t_{0}}(\gamma)$, which is a segment of the same length and parallel to $\gamma$, must contain one endpoint of $\gamma$, without loss of generality, we can assume that $p_{1}$ is contained in $\Psi_{t_{0}}(\gamma) \cap \gamma$. By the definition of $t_{0}$, we have an isometric immersion $\Phi$ from the rectangle $R=[0 ;|\gamma|] \times\left[0 ; t_{0}\right] \subset \mathbb{R}^{2}$ into $\Sigma$ whose restriction into $\operatorname{int}(R)$ is an embedding. We can suppose that $\Phi$ maps the lower side of $R$ onto $\gamma$. Then $\Phi^{-1}\left(\left\{p_{1}\right\}\right)=\left\{\widetilde{p}_{1}^{\prime}, \widetilde{p}_{1}^{\prime \prime}\right\}$, where $\widetilde{p}_{1}^{\prime}$ is an endpoint of the lower side of $R$, and $\widetilde{p}_{1}^{\prime \prime}$ is a point in the upper side of $R$. It is easy to see that $\Phi$ sends the segment in $R$ joining $\widetilde{p}_{1}^{\prime}$ and $\tilde{p}_{1}^{\prime \prime}$ to a simple closed geodesic which meets $\gamma$ only at $p_{1}$, and the lemma follows.

Case $\mathcal{H}(2)$ Let $p$ denote the unique singularity of $\Sigma$, and $\tau$ the hyperelliptic involution of $\Sigma$. In this case $\gamma$ is a geodesic segment joining $p$ to itself, and invariant under $\tau$. Note that $\tau$ reverse the orientation of $\gamma$, and since $\tau(p)=p$, it also fixes the midpoint of $\gamma$. 
We can assume that $\gamma$ is horizontal. As before, let $\Psi_{t}, t \in \mathbb{R}$, denote the vertical flow on $\Sigma$. The same argument as in the previous case shows that we have an immersion $\Phi$ from a rectangle $R \subset \mathbb{R}^{2}$ into $\Sigma$ such that $\Phi_{\mid \operatorname{int}(R)}$ is an embedding, $\Phi$ maps the lower side of $R$ onto $\gamma$, and there exists a point $\tilde{p}$ in the upper side of $R$ which is mapped to $p$. Let $\widetilde{\Delta}$ denote the triangle whose vertices are $\widetilde{P}$ and the two endpoints of the lower side of $R$. Since $\widetilde{\Delta}$ is contained in $R$, the restriction $\left.\Phi\right|_{\operatorname{int}(\tilde{\Delta})}$ is an embedding, moreover the images of the sides of $\tilde{\Delta}$ by $\Phi$ are three distinct saddle connections, which meet one another only at $p$. Therefore, $\Delta=\Phi(\tilde{\Delta})$ is an embedded triangle in $\Sigma$ whose vertices coincide with $p$. By construction, $\gamma$ is a side of $\Delta$, let $\sigma_{1}, \sigma_{2}$ denote the two other sides. Let $\Delta^{\prime}, \sigma_{1}^{\prime}, \sigma_{2}^{\prime}$ denote the images of $\Delta, \sigma_{1}, \sigma_{2}$ under $\tau$ respectively. Observe that $\Delta^{\prime}$ is also an embedded triangle in $\Sigma$, and $\gamma$ is a common side of $\Delta^{\prime}$ and $\Delta$. Here, we have two possibilities:

(1) $\Delta$ and $\Delta^{\prime}$ have another common side other than $\gamma$, that is, either $\sigma_{1}^{\prime}=\sigma_{1}$, or $\sigma_{2}=\sigma_{2}^{\prime}$. In this case $\Delta \cup \Delta^{\prime}$ is a simple cylinder, and we are done.

(2) $\gamma$ is the only common side of $\Delta$ and $\Delta^{\prime}$. In this case, $\Delta \cup \Delta^{\prime}$ is an embedded parallelogram in $\Sigma$. Let us show that $\sigma_{1}$ and $\sigma_{1}^{\prime}$ bound a cylinder disjoint from $\Delta \cup \Delta^{\prime}$. Recall the cone angle at $p$ is $6 \pi$, and the action of $\tau$ at $p$ is the rotation of angle $3 \pi$. Fix an orientation for $\gamma$, consider $\gamma$ as a part of $\partial \Delta$ (resp. $\partial \Delta^{\prime}$ ), we then have an orientation for $\sigma_{1}, \sigma_{2}$ (resp. $\sigma_{1}^{\prime}, \sigma_{2}^{\prime}$ ) subsequently. Consider a small disk D centered at $p$. The intersection of any oriented saddle connection with $\mathrm{D}$ is the union of an outgoing ray, and an incoming ray. These two rays specify a pair of angles at $p$, since $\Sigma$ is a translation surface, this pair of angles is either $(\pi, 5 \pi)$, or $(3 \pi, 3 \pi)$. Since $\gamma$ is invariant under $\tau$, the pair of angle specified by $\gamma$ is $(3 \pi, 3 \pi)$, meanwhile the pair of angles specified by $\sigma_{1}$ is $(\pi, 5 \pi)$ since $\sigma_{1}^{\prime}=\tau\left(\sigma_{1}\right) \neq \sigma_{1}$.

We claim that the outgoing and the incoming rays of $\sigma_{1}$ are contained in the same half disk cut out by the outgoing and the incoming rays of $\gamma$. Indeed, suppose that the outgoing and the incoming rays of $\sigma_{1}$ do not belong to the same half disk (see Figure 2, Case (a)). Then by considering the sum of the angles in $\Delta$, we see that the pair of angles specified by $\sigma_{2}$ is $(3 \pi, 3 \pi)$, which means that $\sigma_{2}=\tau\left(\sigma_{2}\right)=\sigma_{2}^{\prime}$, but this is excluded by the hypothesis.

We know that the action of $\tau$ on $H_{1}(\Sigma, \mathbb{Z})$ is - Id, which implies that $\sigma_{1}-\sigma_{1}^{\prime}=0$ in $H_{1}(\Sigma, \mathbb{Z})$. It follows that $\sigma_{1}$ and $\sigma_{1}^{\prime}$ cut $\Sigma$ into two connected components, each of which is equipped with a flat metric structure with piecewise geodesic boundary. Consider the connected component which does not contain $\gamma$. This component does not contain any singularity in its interior, and since the angle between the two rays of $\sigma_{1}$ at $P$ measured inside this component is $\pi$, we deduce that there is no singularities in its boundary. The only flat surface with two geodesic boundary components with 


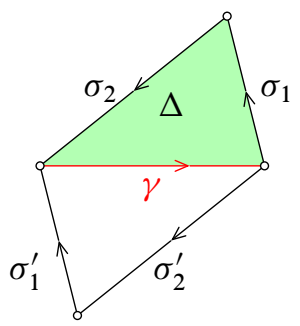

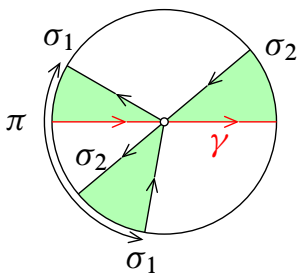

Case (a)

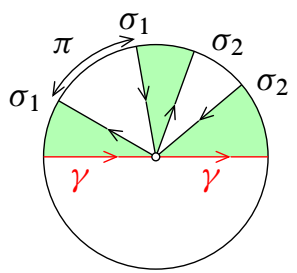

Case (b)

Figure 2: Configurations of geodesic rays at $p$

no singularities is a cylinder. Therefore, we can conclude that $\sigma_{1}$ and $\sigma_{1}^{\prime}$ bound a cylinder $C$ disjoint from $\Delta \cup \Delta^{\prime}$.

Consider the subsurface $\Sigma^{\prime}=\Delta \cup \Delta^{\prime} \cup C$ of $\Sigma$. We first observe that $\Sigma^{\prime}$ is invariant under $\tau$. Topologically, $\Sigma^{\prime}$ is the complement in a torus of two open disks whose boundaries meet at one point. We can construct $\Sigma^{\prime}$ by gluing two parallelograms so that the restriction of $\tau$ into $\Sigma^{\prime}$ is realized by the central symmetries in both parallelograms. Elementary geometry shows that one can find a saddle connection $\eta^{+}$in $\Delta \cup C$ which

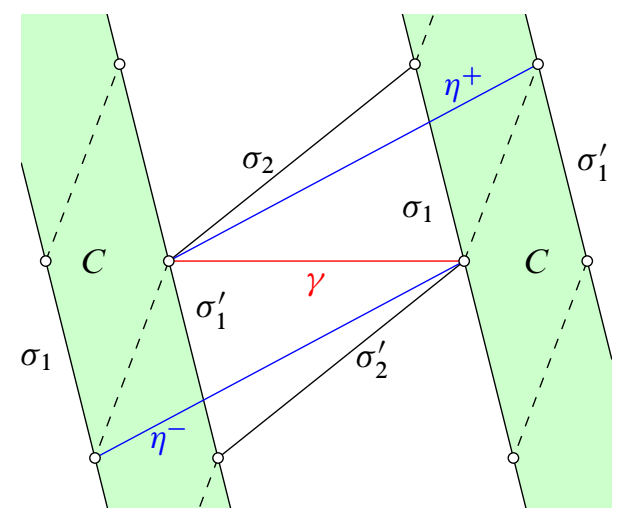

Figure 3: Existence of $\eta^{ \pm}$

crosses $\sigma_{1}$ once (see Figure 3). Let $\eta^{-}$denote the image of $\eta^{+}$under $\tau$. Then $\eta^{+}$ and $\eta^{-}$bound a simple cylinder containing $\gamma$. The lemma is then proved for this case.

Case $\mathcal{H}(1,1)$ Let $\left\{p_{1}, p_{2}\right\}$ denote the singularities of $\Sigma$, the cone angles at both $p_{1}$ and $p_{2}$ are $4 \pi$. Recall that in this case, the hyperelliptic involution $\tau$ exchanges $p_{1}$ and $p_{2}$, therefore $\gamma$ must be a saddle connection joining $p_{1}$ to $p_{2}$. Without loss of generality, we can assume that $\gamma$ is horizontal. As we have seen in the previous cases, there exists an embedded triangle $\Delta$ in $\Sigma$ bounded by $\gamma$ and two other saddle 
connections $\sigma_{1}$ and $\sigma_{2}$. Since there are two singularities, one of the two sides $\sigma_{1}$ and $\sigma_{2}$ must joint a singularity to itself, therefore we can assume that $\sigma_{1}$ joins $p_{1}$ to itself.

Let $\Delta^{\prime}, \sigma_{1}^{\prime}, \sigma_{2}^{\prime}$ denote the images of $\Delta, \sigma_{1}, \sigma_{2}$ under $\tau$ respectively. Since $\tau$ exchanges $p_{1}$ and $p_{2}, \sigma_{1}$ and $\sigma_{1}^{\prime}$ are two distinct saddle connections. We choose the orientation for $\gamma$ to be from $p_{1}$ to $p_{2}$, and choose the orientation of $\sigma_{1}$ and $\sigma_{2}$ (resp. $\sigma_{1}^{\prime}$ and $\sigma_{2}^{\prime}$ ) subsequently to get an orientation for the boundary of $\Delta$ (resp. $\Delta^{\prime}$ ). Consider two small disks $\mathrm{D}_{1}, \mathrm{D}_{2}$ centered at $p_{1}, p_{2}$ respectively. The intersection of $\sigma_{1}$ with $\mathrm{D}_{1}$ consists of an outgoing ray and an incoming ray, while the intersection of $\gamma$ with $\mathrm{D}_{1}$ consists of only an out going ray. Let $\theta$ be the angle between the outgoing and the incoming rays of $\sigma_{1}$ measured along the sector of $\mathrm{D}_{1}$ that does not meet $\gamma$ (see Figure 4).
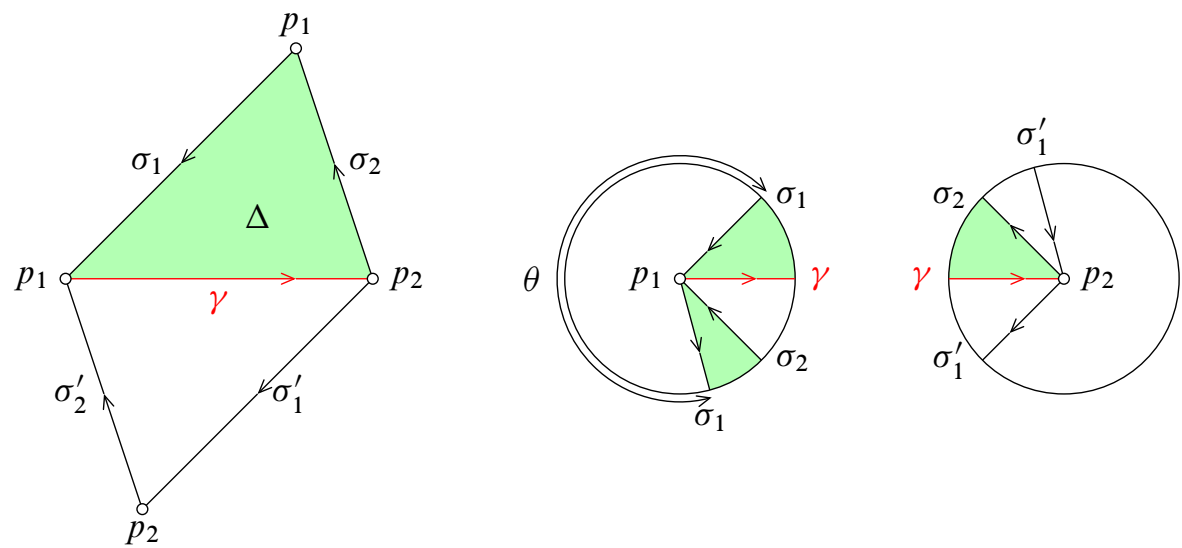

Figure 4: Configuration of geodesic rays at $p_{1}$ and $p_{2}$

We have two cases:

$\theta=3 \pi$ : In this case, the angle between the two rays of $\sigma_{1}$ measured along the sector of $\mathrm{D}_{1}$ which meets $\gamma$ is $\pi$. A simple computation of angles shows that we must have $\sigma_{2}=\sigma_{2}^{\prime}$ as subset of $\Sigma$, which implies that $\Delta \cup \Delta^{\prime}$ is actually a cylinder invariant under $\tau$ and bounded by $\sigma_{1}$ and $\sigma_{1}^{\prime}$, and the lemma follows immediately.

$\theta=\pi$ : Since $\sigma_{1}-\sigma_{1}^{\prime}=0$ in $H_{1}(\Sigma, \mathbb{Z})$, by cutting $\Sigma$ along $\sigma_{1}$ and $\sigma_{1}^{\prime}$, we obtain two flat surfaces with piecewise geodesic boundary. Observe that the component which does not contain $\gamma$ has no singularities in the interior, and since the angle between the two rays of $\sigma_{1}$ measured inside this surface is $\pi$, we see that it has no singularities in the boundary. It follows that this component is a cylinder $C$ bounded by $\sigma_{1}$ and $\sigma_{1}^{\prime}$. Now, using the same argument as in the case $\mathcal{H}(2)$, we see that there exists a pair of saddle connections $\eta^{ \pm}$in $\Delta \cup \Delta^{\prime} \cup C$, which are exchanged by $\tau$, and bound a simple cylinder containing $\gamma$. The proof of the lemma is now complete. 


\section{Proof of Theorem 1.1}

\subsection{Existence of simple cylinder on hyperelliptic translation surfaces}

To prove Theorem 1.1, we first show:

Lemma 3.1 For any $g \geqslant 2$, on every surface of the stratum $\mathcal{H}^{\text {hyp }}(2 g-2)$, there always exists a simple cylinder which is invariant under the hyperelliptic involution.

Proof Let $\Sigma$ be a surface in the stratum $\mathcal{H}^{\text {hyp }}(2 g-2)$. A construction due to Veech [14] (see also [7]) allows us to construct $\Sigma$ from a $2 g$-gon P in $\mathbb{R}^{2}$ centered at the origin, and invariant under the central symmetry of $\mathbb{R}^{2}$. The polygon $\mathrm{P}$ is not necessarily convex, however it has a horizontal diagonal $d$ which passes through the origin contained in the interior. Let $A_{0}, B_{0}$ denote the left and right endpoints of $d$ respectively. We denote by $A_{1}, \ldots, A_{2 g-1}$ (resp. $B_{1}, \ldots, B_{2 g-1}$ ) the vertices of $\mathrm{P}$ above (resp. below) the diagonal $d$ in the counterclockwise order. We consider by convention that $A_{2 g}=B_{0}$, and $B_{2 g}=A_{0}$. The surface $\Sigma$ is obtained by identifying the opposite sides of $\mathrm{P}$ (see Figure 5).

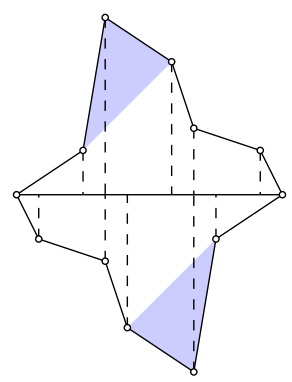

Figure 5: Construction of a hyperelliptic surface from a symmetric polygon

Let $y: \mathbb{R}^{2} \longrightarrow \mathbb{R}$ denote the vertical coordinate function of $\mathbb{R}^{2}$. Let $i_{0}$ be the smallest index in $\{0,1, \ldots, 2 g-1\}$ so that $y\left(A_{i_{0}}\right)=\max \left\{y\left(A_{1}\right), \ldots, y\left(A_{2 g-1}\right)\right\}$. By the choice of $i_{0}$, we see that the diagonal $\overline{A_{i_{0}-1} A_{i_{0}+1}}$ is contained inside P. By symmetry, the diagonal $\overline{B_{i_{0}-1} B_{i_{0}+1}}$ is also contained inside P. Since the sides $\overline{A_{i_{0}-1} A_{i_{0}}}$ and $\overline{A_{i_{0}} A_{i_{0}+1}}$ are identified with $\overline{B_{i_{0}-1} B_{i_{0}}}$ and $\overline{B_{i_{0}} B_{i_{0}+1}}$ respectively, it follows that the union of the two triangles $\Delta^{u}=\left(A_{i_{0}-1} A_{i_{0}} A_{i_{0}+1}\right)$ and $\Delta^{l}=\left(B_{i_{0}-1} B_{i_{0}} B_{i_{0}+1}\right)$ is projected to a simple cylinder $C$ of $\Sigma$. Now, the hyperelliptic involution of $\Sigma$ corresponds to the central symmetry at the origin, which interchanges the two triangles $\Delta^{u}$ and $\Delta^{l}$, therefore the hyperelliptic involution preserves $C$, and exchanges its boundary components.

Remark This lemma is also true for surfaces in $\mathcal{H}^{\text {hyp }}(g-1, g-1)$. 


\subsection{Proof of Theorem 1.1}

Let $\Sigma$ be a surface in $\mathcal{H}^{\text {hyp }}(4)$, we denote by $\tau$ the hyperelliptic involution of $\Sigma$. By Lemma 3.1, we know that there exists a simple cylinder $C_{1}$ in $\Sigma$ bounded by a pair of saddle connections $\left(\delta_{1}^{+}, \delta_{1}^{-}\right)$such that $\tau\left(C_{1}\right)=C_{1}$ and $\tau\left(\delta_{1}^{+}\right)=\delta_{1}^{-}$. Cutting off $C_{1}$ from $\Sigma$, we then get a surface whose boundary is a figure eight, ie the union of two circles meeting at one point. Splitting the common point of the two circles into two points, we get a surface whose boundary is the union of two geodesic segments corresponding to the pair $\left(\delta_{1}^{+}, \delta_{1}^{-}\right)$. Gluing these two segments together, we then get a surface $\Sigma^{\prime}$ in $\mathcal{H}(1,1)$ with a marked saddle connection which will be denoted by $\delta_{1}$.

We consider $\Sigma^{\prime}$ as a subsurface of $\Sigma$. Since $\tau$ preserves $C_{1}$, its restriction $\tau^{\prime}$ to $\Sigma^{\prime}$ is the hyperelliptic involution of $\Sigma^{\prime}$. Observe that $\delta_{1}$ is invariant under $\tau^{\prime}$. By Lemma 2.1, we know that there exists a pair of saddle connections $\left(\delta_{2}^{+}, \delta_{2}^{-}\right)$which bound a simple cylinder $C_{2}$ containing $\delta_{1}$. Again, we have that $\tau^{\prime}$ preserves $C_{2}$ and exchanges $\delta_{2}^{+}$ and $\delta_{2}^{-}$. Note that since $\delta_{2}^{+}$and $\delta_{2}^{-}$meet $\delta_{1}$ at only the endpoints of $\delta_{1}$, which are the singularities of $\Sigma^{\prime}$, we deduce that $\delta_{2}^{+}$and $\delta_{2}^{-}$correspond to a pair of homologous saddle connections in the initial surface $\Sigma$.

Now, cut off $C_{2}$ from $\Sigma^{\prime}$, what is left is a surface with two boundary components corresponding to $\delta_{2}^{+}$and $\delta_{2}^{-}$. Gluing the two boundary components so that the two singularities are identified, we get a surface in $\mathcal{H}(2)$ with a marked saddle connection, which is invariant by the hyperelliptic involution. Lemma 2.1 then allows us to continue the procedure until we are left with a simple cylinder. Since in each step, we cut out a simple cylinder, a simple computation on Euler character shows that we get to this situation after four steps. Thus, this procedure provides us with four pairs of homologous saddle connections $\left(\delta_{i}^{+}, \delta_{i}^{-}\right), i=1, \ldots, 4$, in $\Sigma$ which satisfy the properties asserted in the statement of the theorem.

Corollary 3.2 There exists on any surface $\Sigma$ in $\mathcal{H}^{\text {hyp }}(4)$ a pair of homologous saddle connections which are exchanged by the hyperelliptic involution, and decompose $\Sigma$ into a union of a surface in $\mathcal{H}(2)$, and a surface in $\mathcal{H}(0,0)$. In both components of this decomposition, this pair of saddle connections corresponds to a saddle connection invariant under the distinguished involution.

Proof Let $\left(\delta_{i}^{+}, \delta_{i}^{-}\right), i=1, \ldots, 4$, be the saddle connections in $\Sigma$ satisfying the properties in Theorem 1.1. One can easily check that both pairs $\left(\delta_{2}^{+}, \delta_{2}^{-}\right)$and $\left(\delta_{3}^{+}, \delta_{3}^{-}\right)$ satisfy the property asserted in the corollary. 


\section{Splitting of surfaces in $\mathcal{H}^{\text {hyp }}(4)$}

\subsection{Flat torus with a marked geodesic segment}

Throughout this paper, by a "flat torus" we will mean a Riemann surface of genus one together with a nonzero holomorphic 1-form. Equivalently, we identify a flat torus with the quotient $\mathbb{C} / \Lambda$, where $\Lambda$ is a lattice isomorphic to $\mathbb{Z} \oplus \mathbb{Z}$. Using this identification, we can associate to any oriented geodesic $s$ in the torus a vector $V(s) \in \mathbb{R}^{2}$. If $u=\left(x_{1}, y_{1}\right)$ and $v=\left(x_{2}, y_{2}\right)$ are two vectors in $\mathbb{R}^{2}$, we set $u \wedge v=x_{1} y_{2}-x_{2} y_{1}$. The following lemma is elementary, but will be useful for us in the sequel.

Lemma 4.1 Let $T$ be a flat torus, and $s$ be a geodesic segment joining two distinct points $p_{1}, p_{2}$ in $T$. Let $c$ be a simple closed geodesic passing through $p_{1}$, not parallel to $s$. Then $p_{1}$ is the unique intersection point of $c$ and $s$ if and only if $|V(s) \wedge V(c)|<\operatorname{Area}(T)$.

Proof Using $\operatorname{SL}(2, \mathbb{R})$, we can assume that $V(c)$ is horizontal and $V(s)$ is vertical. Cutting $T$ along $c$, we then get a cylinder $C$. Let $h$ be the height of $C$. The fact that $p_{1}$ is the unique intersection point of $s$ and $c$ is equivalent to $|V(s)|<h$. Since

$$
|V(s) \wedge V(c)|=|V(s)||V(c)|<h|V(c)|=\operatorname{Area}(C)=\operatorname{Area}(T),
$$

the lemma follows immediately.

\subsection{The space of splittings}

Let $\Sigma$ be a surface in $\mathcal{H}^{\text {hyp }}(4)$. We denote by $p$ the unique singularity of $\Sigma$. Let $\delta_{i}^{ \pm}$, $i=1, \ldots, 4$, be four pairs of saddle connections in $\Sigma$ as in Theorem 1.1. Cutting $\Sigma$ along $\left(\delta_{1}^{+}, \delta_{1}^{-}\right)$and $\left(\delta_{3}^{+}, \delta_{3}^{-}\right)$, we get three following components:

- $C_{1}$ is a cylinder bounded by $\delta_{1}^{+}$and $\delta_{1}^{-}$. Gluing $\delta_{1}^{+}$and $\delta_{1}^{-}$together so that the two points corresponding to $p$ are identified, we then get a surface in $\mathcal{H}(0)$ with a marked saddle connection.

- $C_{2}$ is an annulus equipped with a flat metric structure with piecewise geodesic boundaries, each boundary component of $C_{2}$ consists of two geodesic segments (corresponding to $\delta_{1}^{+} \cup \delta_{3}^{+}$, and $\delta_{1}^{-} \cup \delta_{3}^{-}$). Gluing $\delta_{1}^{+}$and $\delta_{3}^{+}$to $\delta_{1}^{-}$and $\delta_{3}^{-}$ respectively, we then get an element of $\mathcal{H}(0,0)$, together with two saddle connections whose union is a simple closed curve.

- $C_{3}$ is a one holed flat torus, the boundary of $C_{3}$ is connected and consists of two geodesic segments corresponding to $\delta_{3}^{+}$and $\delta_{3}^{-}$. Gluing these two segments together, we then get an element in $\mathcal{H}(0,0)$ together with a marked saddle connection. 
Remark We get a similar decomposition of $\Sigma$ by cutting along the pairs $\left(\delta_{2}^{+}, \delta_{2}^{-}\right)$ and $\left(\delta_{4}^{+}, \delta_{4}^{-}\right)$.

Let $\mathbf{S p l}$ denote the set of $\left(T_{1}, T_{2}, T_{3}, v_{1}, v_{2}\right)$, where $T_{1} \in \mathcal{H}(0), T_{2}, T_{3} \in \mathcal{H}(0,0)$ and $v_{i} \in \mathbb{R}^{2}, i=1,2$, satisfy the following:

(a) There are a saddle connection in $T_{1}$ and a saddle connection in $T_{2}$ both have associated vector equal to $v_{1}$.

(b) There are a saddle connection in $T_{2}$, and a saddle connection in $T_{3}$ both have associated vector equal to $v_{2}$.

(c) $v=v_{1}+v_{2}$ is a primitive vector of the lattice $\Lambda_{2}=\Lambda\left(T_{2}\right)$ associated to $T_{2}$, and there exists another primitive vector $w$ such that $\Lambda_{2}=\mathbb{Z} v \oplus \mathbb{Z} w$ and $0<\left|v_{i} \wedge w\right|<\operatorname{Area}\left(T_{2}\right), i=1,2$.

We denote by $\mathbf{S p l}_{1}$ the subset of $\mathbf{S p l}$ consisting of elements $\left(T_{1}, T_{2}, T_{3}, v_{1}, v_{2}\right)$ such that $\operatorname{Area}\left(T_{1}\right)+\operatorname{Area}\left(T_{2}\right)+\operatorname{Area}\left(T_{3}\right)=1$.

Remark - We have a natural action of $\mathrm{GL}^{+}(2, \mathbb{R})$ on Spl.

- Condition (c) implies in particular that, if we denote by $s_{1}$ and $s_{2}$ the saddle connections in $T_{2}$ corresponding $v_{1}$ and $v_{2}$ respectively, then there exists a pair of simple closed geodesics $c^{+}, c^{-}$passing through the two marked points such that $V\left(c^{+}\right)=V\left(c^{-}\right)$, and $c^{ \pm}$cut $T_{2}$ into two cylinders, each of which contains one of the two saddle connections $s_{1}, s_{2}$.

Given an element $\left(T_{1}, T_{2}, T_{3}, v_{1}, v_{2}\right)$ in $\mathbf{S p l}$, we construct a surface in $\mathcal{H}^{\text {hyp }}(4)$ as follows:

- Cutting $T_{1}$ along the saddle connection corresponding to $v_{1}$, we get a cylin$\operatorname{der} C_{1}$.

- Let $s_{1}, s_{2}$ be the saddle connections in $T_{2}$ corresponding to $v_{1}$ and $v_{2}$ respectively. Since $v_{1}+v_{2}$ is a primitive vector in $\Lambda\left(T_{2}\right)$, we see that $s_{1} \cup s_{2}$ is a simple closed curve in $T_{2}$. Cutting $T_{2}$ along $s_{1}$ and $s_{2}$, we then get a cylinder with piecewise geodesic boundary, which will be denoted by $C_{2}$.

- Slitting open $T_{3}$ along saddle connection corresponding to $v_{2}$, we get a one holed torus which will be denoted by $C_{3}$.

- We can now glue $C_{1}, C_{2}, C_{3}$ together following the model shown in Figure 1 so that all the marked points are identified, we then get a surface in $\mathcal{H}^{\text {hyp }}(4)$. 
This construction provides us with a map $\Psi: \mathbf{S p l} \longrightarrow \mathcal{H}^{\text {hyp }}(4)$. A direct consequence of Theorem 1.1 is the following:

Proposition 4.2 The map $\Psi$ is surjective, locally homeomorphic, and $\mathrm{GL}^{+}(2, \mathbb{R})-$ equivariant.

\subsection{Special splitting}

Let $X=\left(T_{1}, T_{2}, T_{3}, v_{1}, v_{2}\right)$ be an element of $\mathbf{S p l , w e ~ s a y ~ t h a t ~} X$ is a special splitting if $v_{1}$ and $v_{2}$ are parallel (collinear). We denote by $\mathbf{S S p l}$ the set of special splittings in $\mathbf{S p l}$, and by $\mathbf{S S p l}_{1}$ the intersection $\mathbf{S S p l} \cap \mathbf{S p l}_{1}$.

Consider a point $X=\left(T_{1}, T_{2}, T_{3}, v_{1}, v_{2}\right)$ in SSpl, we denote by $\Lambda_{i}, i=1,2,3$, the lattices associated to $T_{i}$. Let $C_{1}$ (resp. $C_{2}$ ) denote the cylinder obtained by cutting $T_{1}$ (resp. $T_{2}$ ) along the saddle connection corresponding to $v_{1}$ (resp. along the union of the saddle connections corresponding to $v_{1}$ and $v_{2}$ ). Let $m_{i}, i=1,2$, denote the modulus of $C_{i}$, we will call $m_{1}$ (resp. $\left.m_{2}\right)$ the modulus of the pair $\left(T_{1}, v_{1}\right)$ (resp. of the pair $\left.\left(T_{2}, v_{1}+v_{2}\right)\right)$. By construction, $C_{1}$ and $C_{2}$ are isometric to two cylinders in the direction $v_{1}$ on the surface $\Sigma=\Psi(X)$. Set

$$
\alpha=\frac{\left|v_{2}\right|}{\left|v_{1}\right|} \quad \text { and } \quad \bar{m}=\frac{m_{1}}{m_{2}} .
$$

Observe that we have the following relation between $\bar{m}$ and $\alpha$ :

$$
\bar{m}=\frac{\operatorname{Area}\left(T_{1}\right)}{\operatorname{Area}\left(T_{2}\right)}(1+\alpha)^{2} .
$$

Since $\alpha$ and the areas of $T_{i}$ are $\operatorname{SL}(2, \mathbb{R})$-invariant, so is $\bar{m}$. We will call $\bar{m}$ the moduli ratio of $\left(T_{1}, T_{2}, T_{3}, v_{1}, v_{2}\right)$.

Using $\operatorname{SO}(2, \mathbb{R})$, we can assume that $C_{1}$ and $C_{2}$ are horizontal. We can also define the twists for $C_{1}$ and $C_{2}$ as follows: let $w_{1}=\left(w_{1}^{x}, w_{1}^{y}\right)\left(\right.$ resp. $\left.w_{2}=\left(w_{2}^{x}, w_{2}^{y}\right)\right)$ be a primitive vector in $\Lambda_{1}$ (resp. $\left.\Lambda_{2}\right)$ such that $\Lambda_{1}=\mathbb{Z} v_{1} \oplus \mathbb{Z} w_{1}$ (resp. $\Lambda_{2}=$ $\left.\mathbb{Z}\left(v_{1}+v_{2}\right) \oplus \mathbb{Z} w_{2}\right)$. We define the twists $t_{1}, t_{2}$ of $C_{1}$ and $C_{2}$ respectively to be

$$
t_{1}=\frac{w_{1}^{x}}{\left|v_{1}\right|} \bmod \mathbb{Z} \quad \text { and } \quad t_{2}=\frac{w_{2}^{x}}{\left|v_{1}\right|+\left|v_{2}\right|} \bmod \mathbb{Z} .
$$

We also call $t_{1}$ (resp. $\left.t_{2}\right)$ the twist of the pair $\left(T_{1}, v_{1}\right)$ (resp. of the pair $\left(T_{2}, v_{1}+v_{2}\right)$ ). Recall that a vector $w$ in $\mathbb{R}^{2}$ is generic with respect to a lattice $\Lambda=\mathbb{Z} u \oplus \mathbb{Z} v$ if $w$ is not parallel to any vector in $\Lambda$. We first prove a slightly weaker version of Theorem 1.2, where we suppose in addition that the moduli ratio is irrational. As we will see, this condition can be relaxed, and we obtain Theorem 1.2 as a consequence of this theorem. 
Theorem 4.3 Let $X_{0}=\left(T_{1}^{0}, T_{2}^{0}, T_{3}^{0}, v_{1}^{0}, v_{2}^{0}\right)$ be an element in $\mathbf{S S p l}_{1}$. Let $\Lambda_{i}^{0}, i=$ $1,2,3$, denote the lattice associated to $T_{i}^{0}$, and $\bar{m}_{0}$ denote the moduli ratio of $X_{0}$. Suppose that

- $\bar{m}_{0} \notin \mathbb{Q}$,

- $v_{2}^{0}$ is generic with respect to $\Lambda_{3}^{0}$.

Then $\mathcal{O}:=\operatorname{SL}(2, \mathbb{R}) \cdot \Psi\left(X_{0}\right)$ is dense in $\mathcal{H}_{1}^{\text {hyp }}(4)$.

\subsection{Ratner's Theorem}

The first important ingredient of the proof of Theorem 4.3 is a consequence of the famous theorem of Ratner on action of unipotent subgroups on homogeneous spaces. Before stating this theorem, let us first recall some basic notions. Let $G$ be a Lie group, and $\mathfrak{g}$ be its Lie algebra. An element $g$ of $G$ is unipotent if $\operatorname{Ad}_{g}-\mathrm{Id}$ is nilpotent in $\operatorname{End}(\mathfrak{g})$. Let $\lambda$ denote the right Haar measure of $G, G$ is called unimodular if the left Haar measure equals the right Haar measure, or equivalently if $\left|\operatorname{det} \operatorname{Ad}_{g}\right|=1$ for all $g$ in $G$. A discrete subgroup $\Gamma$ of $G$ is called a lattice if we have $\lambda(G / \Gamma)<\infty$. If $G$ has a lattice then it is unimodular. It is well-known that $\operatorname{SL}(2, \mathbb{R})$ is unimodular, but its subgroup consisting upper triangular matrices is not.

Theorem 4.4 (Ratner) Let $G$ be a finite dimensional Lie group, $\Gamma$ be a lattice in $G$, and $X=G / \Gamma$. Let $U$ be a connected subgroup of $G$ generated by unipotent element. Then for any $x$ in $X$, the closure $\overline{U \cdot x}$ of the $U$-orbit of $x$ is a homogeneous space of finite volume, that is there exists a closed unimodular subgroup $H \subset G$ containing $U$ such that

- $\overline{U \cdot x}=H \cdot x$,

- $x \Gamma x^{-1} \cap H$ is a lattice in $H$.

Put $G=\mathbb{R} \times \mathbb{R} \times \operatorname{SL}(2, \mathbb{R})$ and $\Gamma=\mathbb{Z} \times \mathbb{Z} \times \operatorname{SL}(2, \mathbb{Z})$. Then $\Gamma$ is a lattice in $G$. An element of $G / \Gamma$ is a triple $\left(\theta_{1}, \theta_{2}, \Lambda\right)$, where $\theta_{i} \in \mathbb{R} / \mathbb{Z} \simeq \mathbb{S}^{1}$, and $\Lambda \simeq \mathbb{Z}^{2}$ is a lattice in $\mathbb{R}^{2}$ such that $\operatorname{Vol}\left(\mathbb{R}^{2} / \Lambda\right)=1$. Let $m_{1}, m_{2}$ be two positive real numbers. We set

$$
U=U_{m_{1}, m_{2}}=\left\{\left(m_{1} t, m_{2} t,\left(\begin{array}{ll}
1 & t \\
0 & 1
\end{array}\right)\right), t \in \mathbb{R}\right\},
$$

then $U$ is a unipotent subgroup of $G$. As we will see, the space $X=G / \Gamma$ can be used to parametrize the subspace of space of special splittings consisting of splittings such that $v_{1}=(1,0), v_{2}=(\alpha, 0), \alpha$ is a constant, and the areas of the tori are fixed. 
In this parametrization, the action of $U_{m_{1}, m_{2}}$ defined above corresponds to the action of the unipotent subgroup

$$
\left(\begin{array}{ll}
1 & t \\
0 & 1
\end{array}\right), \quad t \in \mathbb{R}
$$

of $\operatorname{SL}(2, \mathbb{R})$. Applying Theorem 4.4 in this case, we have:

Corollary 4.5 Suppose that $m_{1} / m_{2} \notin \mathbb{Q}$. Let $\Lambda$ be a lattice in $\mathbb{R}^{2}$ which contains no horizontal vectors. Then for any $\left(\theta_{1}, \theta_{2}\right) \in \mathbb{R} / \mathbb{Z} \times \mathbb{R} / \mathbb{Z}$, we have

$$
\overline{U \cdot\left(\theta_{1}, \theta_{2}, \Lambda\right)}=G / \Gamma \text {. }
$$

Proof By Ratner Theorem, we know that $\overline{U \cdot\left(\theta_{1}, \theta_{2}, \Lambda\right)}=H \cdot\left(\theta_{1}, \theta_{2}, \Lambda\right)$, where $H$ is connected, unimodular subgroup of $G$. All we need to show is that $H=G$.

Let $x$ be any element of $G$ which is projected to $\left(\theta_{1}, \theta_{2}, \Lambda\right)$. Let $\mathfrak{h}$ and $\mathfrak{g}$ denote the Lie algebras of $H$ and $G$ respectively. Set

$$
\mathbf{a}=\left(\begin{array}{cc}
1 & 0 \\
0 & -1
\end{array}\right), \quad \mathbf{u}_{+}=\left(\begin{array}{ll}
0 & 1 \\
0 & 0
\end{array}\right), \quad \mathbf{u}_{-}=\left(\begin{array}{ll}
0 & 0 \\
1 & 0
\end{array}\right) .
$$

We have $\mathfrak{s l}(2, \mathbb{R})=\mathbb{R} \mathbf{u}_{+} \oplus \mathbb{R} \mathbf{u}_{-} \oplus \mathbb{R} \mathbf{a}$, and $\mathfrak{g}=\mathbb{R} \oplus \mathbb{R} \oplus \mathfrak{s l}(2, \mathbb{R})$. Observe that the Lie bracket of $\mathfrak{g}$ is trivial on the $\mathbb{R}$ components, and we have

$$
\left[\mathbf{u}_{+}, \mathbf{u}_{-}\right]=\mathbf{a},\left[\mathbf{a}, \mathbf{u}_{+}\right]=2 \mathbf{u}_{+},\left[\mathbf{a}, \mathbf{u}_{-}\right]=-2 \mathbf{u}_{-} .
$$

Since $U \subset H$, the Lie algebra $\mathfrak{h}$ contains $\mathbf{v}_{0}=\left(m_{1}, m_{2}, \mathbf{u}_{+}\right)$. Observe that $x \Gamma x^{-1}=$ $\mathbb{Z} \times \mathbb{Z} \times M \cdot \operatorname{SL}(2, \mathbb{Z}) \cdot M^{-1}$, where $M$ is any matrix in $\operatorname{SL}(2, \mathbb{R})$ sending the standard basis of $\mathbb{R}^{2}$ to a basis of the lattice $\Lambda$. We denote by $A$ and $N$ be the following subgroups of $\operatorname{SL}(2, \mathbb{R})$ :

$$
A=\left\{\left(\begin{array}{cc}
e^{t} & 0 \\
0 & e^{-t}
\end{array}\right), t \in \mathbb{R}\right\}, \quad N=\left\{\left(\begin{array}{ll}
1 & t \\
0 & 1
\end{array}\right), t \in \mathbb{R}\right\} .
$$

Let $\mathrm{pr}_{2}: \mathfrak{g} \longrightarrow \mathfrak{s l}(2, \mathbb{R})$ denote the natural projection. The image of $\mathfrak{h}$ under $\mathrm{pr}_{2}$ is a subalgebra of $\mathfrak{s l}(2, \mathbb{R})$ which contains $\mathbb{R} \mathbf{u}_{+}$.

Case $1 \operatorname{pr}_{2}(\mathfrak{h})=\mathbb{R} \mathbf{u}_{+}$. We have three possibilities:

- $\mathfrak{h}=\mathbb{R} \mathbf{v}_{0}$ implies $H=U$, but by assumption, $U \cap \mathbb{Z} \times \mathbb{Z} \times M \cdot \operatorname{SL}(2, \mathbb{Z}) \cdot M^{-1}=$ $\{(0,0, \mathrm{Id})\}$ is not a lattice in $U$.

- $\mathfrak{h}=\mathbb{R} \mathbf{v}_{0} \oplus \mathbb{R} \mathbf{u}_{+}=\mathbb{R} \mathbf{u}_{+} \oplus \mathbb{R}_{m_{1}, m_{2}}$, where $\mathbb{R}_{m_{1}, m_{2}}=\mathbb{R} \cdot\left(m_{1}, m_{2}\right) \subset \mathbb{R}^{2}$. It follows that $H=\mathbb{R}_{m_{1}, m_{2}} \times N$. But again, $H \cap \mathbb{Z} \times \mathbb{Z} \times M \cdot \operatorname{SL}(2, \mathbb{Z}) \cdot M^{-1}=$ $\{(0,0, \mathrm{Id})\}$.

- $\mathfrak{h}=\mathbb{R}^{2} \oplus \mathbb{R} \mathbf{u}_{+} \Longrightarrow H=\mathbb{R}^{2} \times N$. But we have $N \cap M \cdot \operatorname{SL}(2, \mathbb{Z}) \cdot M^{-1}=\{\operatorname{Id}\}$, therefore, $H \cap \mathbb{Z} \times \mathbb{Z} \times M \cdot \operatorname{SL}(2, \mathbb{Z}) \cdot M^{-1}$ is not a lattice. 
Case $2 \operatorname{pr}_{2}(\mathfrak{h})=\mathbb{R} \mathbf{u}_{+} \oplus \mathbb{R} \mathbf{a}$. Let $v$ be any vector in $\mathfrak{h}$ such that $\operatorname{pr}_{2}(\mathbf{v})=\mathbf{a}$. Then we have $\left[\mathbf{v}, \mathbf{v}_{0}\right]=2 \mathbf{u}_{+}$. Therefore, we see that $\mathfrak{h}$ contains the vectors

- $\mathbf{u}_{+}$,

- $\mathbf{v}_{1}=\mathbf{a}+\mathbf{w}$, with $\mathbf{w}=\left(k_{1}, k_{2}\right) \in \mathbb{R}^{2}$,

- $\mathbf{w}_{0}=\mathbf{v}_{\mathbf{0}}-\mathbf{u}_{+}=\left(m_{1}, m_{2}\right) \in \mathbb{R}^{2}$.

Here we have two possibilities:

- $\mathfrak{h}=\mathbb{R} \mathbf{w}_{0} \oplus \mathbb{R} \mathbf{u}_{+} \oplus \mathbb{R} \mathbf{v}_{1} \Longrightarrow H=\mathbb{R} \times A^{\prime} N$, where

$$
A^{\prime}=\left\{\left(k_{1} t, k_{2} t,\left(\begin{array}{cc}
e^{t} & 0 \\
0 & e^{-t}
\end{array}\right)\right), t \in \mathbb{R}\right\} \subset G .
$$

It follows that $H \simeq \mathbb{R} \times A N$. But since $A N$ is not unimodular, neither is $H$.

- $\mathfrak{h}=\mathbb{R}^{2} \oplus \mathbb{R} \mathbf{u}_{+} \oplus \mathbb{R} \mathbf{a}$, therefore $H=\mathbb{R}^{2} \times A N$, but again $H$ is not unimodular.

Case $3 \operatorname{pr}_{2}(\mathfrak{h})=\mathfrak{s l}(2, \mathbb{R})$. Let $\mathbf{v}$ be a vector in $\mathfrak{h}$ such that $\operatorname{pr}_{2}(\mathbf{v})=\mathbf{u}_{-}$. Then

$$
\left[\mathbf{v}_{0}, \mathbf{v}\right]=\mathbf{a},\left[\left[\mathbf{v}_{0}, \mathbf{v}\right], \mathbf{v}_{0}\right]=2 \mathbf{u}_{+} \text {and }\left[\left[\mathbf{v}_{0}, \mathbf{v}\right], \mathbf{v}\right]=-2 \mathbf{u}_{-} .
$$

It follows that $\mathfrak{s l}(2, \mathbb{R}) \subset \mathfrak{h}$. We then have two possibilities:

- $\quad H=\mathbb{R}_{m_{1}, m_{2}} \times \operatorname{SL}(2, \mathbb{R})$, in this case $H \cap \mathbb{Z} \times \mathbb{Z} \times M \cdot \operatorname{SL}(2, \mathbb{Z}) \cdot M^{-1}=$ $\left(0,0, M \cdot \operatorname{SL}(2, \mathbb{Z}) \cdot M^{-1}\right)$ is not a lattice in $H$.

- $H=\mathbb{R}^{2} \times \operatorname{SL}(2, \mathbb{R})$, this is the only admissible possibility.

We can then conclude that $\overline{U \cdot\left(\theta_{1}, \theta_{2}, \Lambda\right)}=G / \Gamma$.

Remark Similar results for $\mathbb{R}^{k} \times \operatorname{SL}(2, \mathbb{R})^{n} / \mathbb{Z}^{k} \times \operatorname{SL}(2, \mathbb{Z})^{n}$ with small $k$ and $n$ can be found in [7].

For any $\left(A_{1}, A_{2}, A_{3}, \alpha\right)$ in $\mathbb{R}_{>0}^{4}$, let $\operatorname{SSpl}\left(A_{1}, A_{2}, A_{3}, \alpha\right)$ denote the subset of $\mathbf{S S p l}$ consisting of elements $\left(T_{1}, T_{2}, T_{3}, v_{1}, v_{2}\right)$ such that $\operatorname{Area}\left(T_{i}\right)=A_{i}, i=1,2,3$, and $\left|v_{2}\right| /\left|v_{1}\right|=\alpha$. Using Corollary 4.5, we have the following lemma:

Lemma 4.6 Let $X=\left(T_{1}, T_{2}, T_{3}, v_{1}, v_{2}\right)$ be an element in $\operatorname{SSpl}\left(A_{1}, A_{2}, A_{3}, \alpha\right)$. If $\left(A_{1} / A_{2}\right)(\alpha+1)^{2}$ is irrational, and $v_{2}$ is generic with respect to the lattice $\Lambda_{3}=\Lambda\left(T_{3}\right)$ then

$$
\Psi\left(\operatorname{SSpl}\left(A_{1}, A_{2}, A_{3}, \alpha\right)\right) \subset \overline{\operatorname{SL}(2, \mathbb{R}) \cdot \Psi(X)} .
$$

In particular, let $X_{0}=\left(T_{1}^{0}, T_{2}^{0}, T_{3}^{0}, v_{1}^{0}, v_{2}^{0}\right)$ be as in Theorem 4.3. Then

$$
\Psi\left(\operatorname{SSpl}\left(A_{1}^{0}, A_{2}^{0}, A_{3}^{0}, \alpha_{0}\right)\right) \subset \overline{\operatorname{SL}(2, \mathbb{R}) \cdot \Psi\left(X_{0}\right)},
$$

where $A_{i}^{0}=\operatorname{Area}\left(T_{i}^{0}\right), i=1,2,3$, and $\alpha_{0}=\left|v_{2}^{0}\right| /\left|v_{1}^{0}\right|$. 
Proof Let $\operatorname{SSpl}\left(A_{1}, A_{2}, A_{3}, \alpha\right)$ hor denote the subset of $\operatorname{SSpl}\left(A_{1}, A_{2}, A_{3}, \alpha\right)$ consisting of elements $\left(T_{1}, T_{2}, T_{3}, v_{1}, v_{2}\right)$ with $v_{1}=(1,0)$. Observe that

$$
\operatorname{SSpl}\left(A_{1}, A_{2}, A_{3}, \alpha\right)=\operatorname{SL}(2, \mathbb{R}) \cdot \operatorname{SSpl}\left(A_{1}, A_{2}, A_{3}, \alpha\right)_{\text {hor }} .
$$

We have a natural mapping $\varphi: \operatorname{SSpl}\left(A_{1}, A_{2}, A_{3}, \alpha\right)_{\text {hor }} \longrightarrow G / \Gamma$ which sends a special splitting $\left(T_{1}, T_{2}, T_{3}, v_{1}, v_{2}\right)$ to an element $\left(t_{1}, t_{2}, \Lambda\right) \in G / \Gamma$, where $t_{1}$ and $t_{2}$ are the twists of $\left(T_{1}, v_{1}\right)$ and $\left(T_{2}, v_{1}+v_{2}\right)$ respectively, and $\Lambda$ is the lattice associated to $T_{3}$ normalized to have covolume one. Remark that $\varphi$ is a homeomorphism onto its image, and that $\varphi\left(\operatorname{SSpl}\left(A_{1}, A_{2}, A_{3}, \alpha\right)_{\text {hor }}\right)$ is an open dense set of $G / \Gamma$, its complement consists of triples $\left(\theta_{1}, \theta_{2}, \Lambda\right)$ where the lattice $\Lambda$ contains a horizontal vector of length $\leqslant \alpha / \sqrt{A_{3}}$ (this set corresponds to the case where $v_{2}=\lambda v$ with $v \in \Lambda\left(T_{3}\right)$, and $\lambda \geqslant 1$ ).

Let $m_{1}$ and $m_{2}$ denote the moduli of $\left(T_{1}, v_{1}\right)$ and $\left(T_{2}, v_{1}+v_{2}\right)$ respectively. Recall that we have $m_{1} / m_{2}=\left(A_{1} / A_{2}\right)(\alpha+1)^{2}$. Clearly, the set $\operatorname{SSpl}\left(A_{1}, A_{2}, A_{3}, \alpha\right)_{\text {hor }}$ is invariant under the action of the group

$$
U=\left\{\left(\begin{array}{ll}
1 & t \\
0 & 1
\end{array}\right), t \in \mathbb{R}\right\} .
$$

We define the action of $U$ on $G / \Gamma$ using the identification $U \simeq U_{m_{1}, m_{2}}$. It follows that $\varphi$ is $U$-equivariant.

Without loss of generality, we can assume that $X \in \operatorname{SSpl}\left(A_{1}, A_{2}, A_{3}, \alpha\right)_{\text {hor }}$. Consider $x=\varphi(X) \in G / \Gamma$. The hypothesis on $X$ implies that $x$ satisfies the conditions of Corollary 4.5, therefore $\overline{U \cdot x}=G / \Gamma$. Since $\varphi$ is $U$-equivariant and a local homeomorphism, we deduce that $\overline{U \cdot X}=\varphi^{-1}(\overline{U \cdot x})=\operatorname{SSpl}\left(A_{1}, A_{2}, A_{3}, \alpha\right)_{\text {hor }}$, and the lemma follows.

\section{Surfaces admitting special splitting are contained in the or- bit closure}

Our aim in this section is to prove the following

Proposition 5.1 Let $X_{0}=\left(T_{1}^{0}, T_{2}^{0}, T_{3}^{0}, v_{1}^{0}, v_{2}^{0}\right)$ be as in Theorem 4.3. We have $\Psi\left(\mathbf{S S p l}_{1}\right) \subset \overline{\operatorname{SL}(2, \mathbb{R}) \cdot \Psi\left(X_{0}\right)}$.

\subsection{Dual splitting}

Given $X=\left(T_{1}, T_{2}, T_{3}, v_{1}, v_{2}\right)$ in $\mathbf{S S p l}$, we will denote both saddle connections in $T_{1}$ and $T_{2}$ corresponding to $v_{1}$ by $\delta_{1}$, similarly, we denote by $\delta_{2}$ the two saddle 
connections in $T_{2}$ and $T_{3}$ corresponding to $v_{2}$. Recall that the saddle connections $\delta_{i}$, $i=1,2$, give rises to a pair of homologous saddle connections in the surface $\Sigma=\Psi(X)$, which will be denoted by $\delta_{i}^{ \pm}$.

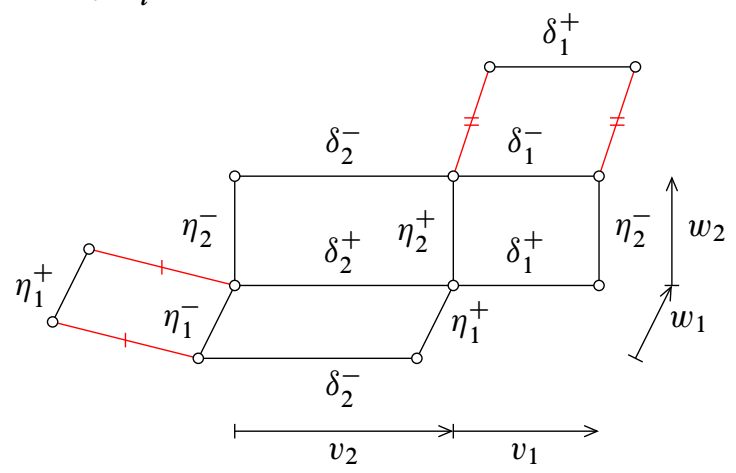

Figure 6: Dual splittings

Let $\eta_{1}$ be a simple closed geodesic in $T_{3}$ which meets $\delta_{2}$ once, and let $\eta_{1}^{ \pm}$denote the pair of saddle connections parallel to $\eta_{1}$. Similarly, let $\eta_{2}$ be a simple closed geodesics in $T_{2}$ which meets $\delta_{1} \cup \delta_{2}$ once, and let $\eta_{2}^{ \pm}$denote the pair of saddle connections parallel to $\eta_{2}$. Observer that $\eta_{1}^{ \pm}$(resp. $\eta_{2}^{ \pm}$) are homologous saddle connections in $\Sigma$. We choose the orientation of $\eta_{1}$ and $\eta_{2}$ so that $\eta_{1}^{+} * \eta_{2}^{+}$is freely homotopic to a simple closed curve in $\Sigma$. Set $w_{1}=V\left(\eta_{1}\right), w_{2}=V\left(\eta_{2}\right)$.

Cutting $\Sigma$ along $\eta_{1}^{ \pm}$and $\eta_{2}^{ \pm}$, we see that the surface $\Sigma$ is obtained from another element $X^{\vee}=\left(T_{1}^{\vee}, T_{2}^{\vee}, T_{3}^{\vee}, w_{1}, w_{2}\right)$ in Spl. We will call $X^{\vee}$ a dual splitting of $X$. Note that $X^{\vee}$ does not belong to SSpl in general, and there are infinitely many splittings dual to a given splitting. We also have

$$
\operatorname{Area}\left(T_{3}^{\vee}\right)=\operatorname{Area}\left(T_{1}\right)+\frac{\operatorname{Area}\left(T_{2}\right)}{1+\left|v_{2}\right| /\left|v_{1}\right|} \operatorname{~}
$$

Throughout this section, we set $A_{i}^{0}=\operatorname{Area}\left(T_{i}^{0}\right), i=1,2,3$, and $\alpha_{0}=\left|v_{2}^{0}\right| /\left|v_{1}^{0}\right|$.

\subsection{Changing splitting}

The first step to prove Proposition 5.1 is the following:

Lemma 5.2 If $\left(A_{1}, A_{2}, A_{3}, \alpha\right) \in \mathbb{R}_{>0}^{4}$ satisfies

$$
\begin{aligned}
A_{1}+A_{2}+A_{3} & =1, \\
A_{1}+\frac{A_{2}}{1+\alpha} & =A_{1}^{0}+\frac{A_{2}^{0}}{1+\alpha_{0}},
\end{aligned}
$$

then $\Psi\left(\operatorname{SSpl}\left(A_{1}, A_{2}, A_{3}, \alpha\right)\right) \subset \overline{\operatorname{SL}(2, \mathbb{R}) \cdot \Psi\left(X_{0}\right)}$. 
Remark We know $\overline{\operatorname{SL}(2, \mathbb{R}) \cdot \Psi\left(X_{0}\right)}$ contains $\Psi\left(\operatorname{SSpl}\left(A_{1}^{0}, A_{2}^{0}, A_{3}^{0}, \alpha_{0}\right)\right)$ by Lemma 4.6. Let $X=\left(T_{1}, T_{2}, T_{3}, v_{1}, v_{2}\right)$ be an element in $\operatorname{SSpl}\left(A_{1}^{0}, A_{2}^{0}, A_{3}^{0}, \alpha_{0}\right)$, and $\Sigma$ be the surface in $\mathcal{H}^{\text {hyp }}(4)$ constructed from $X$. Let $\delta_{i}, \delta_{i}^{ \pm}, \eta_{i}, \eta_{i}^{ \pm}, i=1,2$, and $X^{\vee}=\left(T_{1}^{\vee}, T_{2}^{\vee}, T_{3}^{\vee}, w_{1}, w_{2}\right)$ be as in the previous subsection, where $X^{\vee}$ is a dual splitting of $X$.

Let $\sigma_{1}^{ \pm}$(resp. $\sigma_{2}^{ \pm}$) be a pair of homologous saddle connections in $T_{3}^{\vee}\left(\right.$ resp. $T_{2}^{\vee}$ ) which bound a simple cylinder containing $\eta_{2}$ (see Figure 7). Viewed as saddle connections of $\Sigma$, the pairs $\sigma_{1}^{ \pm}$and $\sigma_{2}^{ \pm}$determine a splitting of $\Sigma$. If $\sigma_{1}^{ \pm}$and $\sigma_{2}^{ \pm}$are parallel, then we have another special splitting of $\Sigma$. To prove the lemma, we will show that for any $\left(A_{1}, A_{2}, A_{3}, \alpha\right)$ in $\mathbb{R}_{>0}^{4}$, there exists an element $X$ in $\operatorname{SSpl}\left(A_{1}^{0}, A_{2}^{0}, A_{3}^{0}, \alpha_{0}\right)$ for which one can find $\sigma_{1}^{ \pm}, \sigma_{2}^{ \pm}$determining a special splitting with parameters $\left(A_{1}, A_{2}, A_{3}, \alpha\right)$. We can then use Lemma 4.6 to conclude, first, for $\left(A_{1}, A_{2}, A_{3}, \alpha\right)$ satisfying the irrational condition of Lemma 4.6, and then for all $\left(A_{1}, A_{2}, A_{3}, \alpha\right)$ by continuity.

Proof of Lemma 5.2 Without loss of generality, we can assume that $v_{1}=(1,0)$ and $v_{2}=\left(\alpha_{0}, 0\right)$. Let $C_{1}$ (resp. $\left.C_{2}\right)$ denote the cylinder obtained by slitting $T_{1}$ (resp. $T_{2}$ ) along saddle connection $\delta_{1}$ (resp. along the saddle connections $\delta_{1}$ and $\delta_{2}$ ). Let $h_{i}$ and $t_{i}$ denote the height and the twist of $C_{i}, i=1,2$. Note that $h_{1}=A_{1}^{0}$, and $h_{2}=A_{2}^{0} /\left(\alpha_{0}+1\right)$. We fix $t_{2}=0$, consequently, we can choose $\eta_{2}^{ \pm}$to be vertical, and therefore $w_{2}=V\left(\eta_{2}^{ \pm}\right)=\left(0, h_{2}\right)$.

Set $\Lambda_{i}=\Lambda\left(T_{i}\right)$ and $\Lambda_{i}^{\vee}=\Lambda\left(T_{i}^{\vee}\right), i=1,2,3$. Recall that $v_{1}$ is a primitive vector of $\Lambda_{1}$, let $u_{1}=\left(x, h_{1}\right)$ be another primitive vector in $\Lambda_{1}$ such that $\Lambda_{1}=\mathbb{Z} u_{1} \oplus \mathbb{Z} v_{1}$. Observe that the parameter $x$ can be chosen arbitrarily. Similarly, $w_{1}=(y, z)$ is a primitive vector in $\Lambda_{1}^{\vee}$, let $\widehat{u}_{1}$ be another primitive vector such that $\Lambda_{1}^{\vee}=\mathbb{Z} w_{1} \oplus \mathbb{Z} \hat{u}_{1}$. Note that $\Lambda_{2}^{\vee}=\mathbb{Z} v_{2} \oplus \mathbb{Z}\left(w_{1}+w_{2}\right)$ and $\Lambda_{3}=\mathbb{Z} w_{1} \oplus \mathbb{Z}\left(v_{2}+\hat{u}_{1}\right)$. The parameters $\left(x, y, z, \widehat{u}_{1}\right) \in \mathbb{R}^{3} \times \mathbb{R}^{2}$ uniquely determine the element $X$ in $\operatorname{SSpl}\left(A_{1}^{0}, A_{2}^{0}, A_{3}^{0}, \alpha_{0}\right)$. By construction, the parameters $\left(x, y, z, \widehat{u}_{1}\right)$ must satisfy the conditions

$$
\begin{aligned}
\left|v_{2} \wedge w_{2}\right|<\operatorname{Area}\left(T_{2}^{\vee}\right)= & \left|v_{2} \wedge\left(w_{1}+w_{2}\right)\right|<1-\operatorname{Area}\left(T_{3}^{\vee}\right)=\frac{\alpha_{0} A_{2}^{0}}{\alpha_{0}+1}+A_{3}^{0}, \\
& \left|w_{1} \wedge\left(v_{2}+\widehat{u}_{1}\right)\right|=A_{3}^{0} .
\end{aligned}
$$

Simple computations show that (2) is equivalent to

$$
0<z<\frac{A_{3}^{0}}{\alpha_{0}} .
$$

Note that the conditions (2) and (3) are sufficient, that is, if the parameters $\left(x, y, z, \widehat{u}_{1}\right)$ satisfy these two conditions, then they determine an element in $\operatorname{SSpl}\left(A_{1}^{0}, A_{2}^{0}, A_{3}^{0}, \alpha_{0}\right)$. 


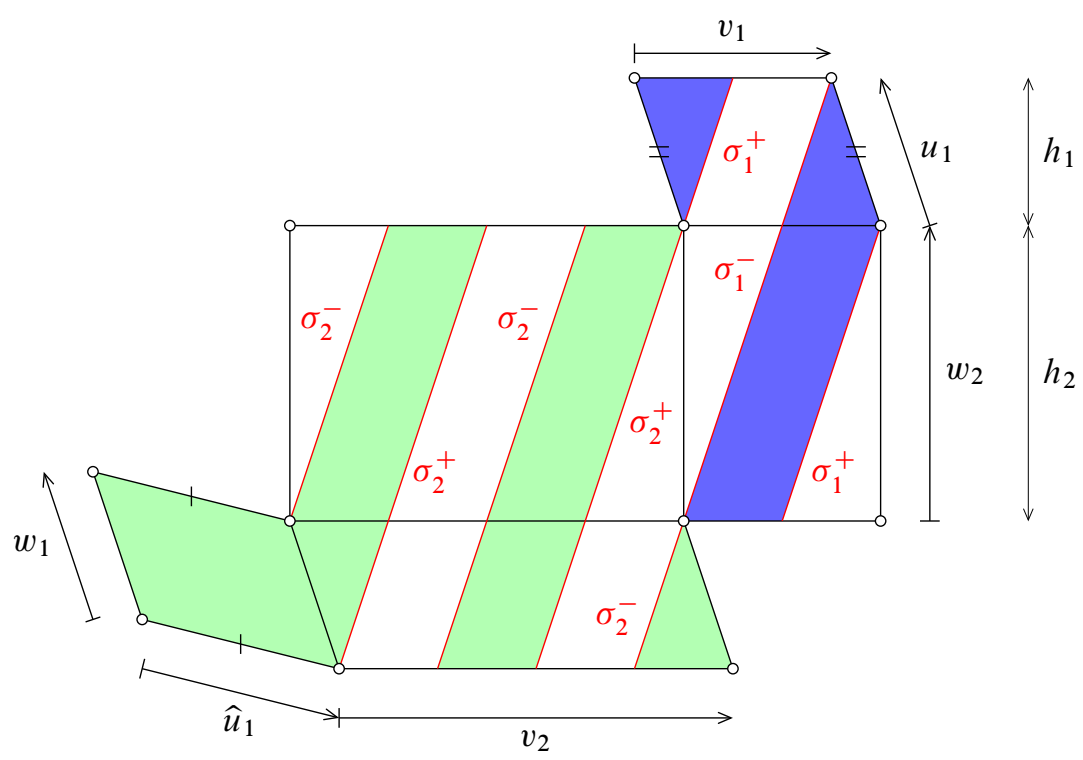

Figure 7: Finding new special splittings

Claim 1 For any $\left(A_{1}, A_{2}, A_{3}, \alpha\right) \in \mathbb{R}_{>0}^{4}$ satisfying the conditions of the lemma, there exist $(x, y, z) \in \mathbb{R}^{3}$ with $z$ satisfying (4) such that we can find a primitive vector $v_{1}^{\prime}$ in $\Lambda_{3}^{\vee}$, and a primitive vector $v_{2}^{\prime}$ in $\Lambda_{2}^{\vee}$ such that

(i) $v_{2}^{\prime}=\alpha v_{1}^{\prime}$,

(ii) $\left|v_{1}^{\prime} \wedge w_{2}\right|=A_{2} /(\alpha+1)$,

(iii) $\left|v_{2}^{\prime} \wedge w_{2}\right|<\operatorname{Area}\left(T_{2}^{\vee}\right)$.

Proof of Claim 1 Recall that, by assumption, we have

$$
A_{1}+\frac{A_{2}}{\alpha+1}=A_{1}^{0}+\frac{A_{2}^{0}}{\alpha_{0}+1} .
$$

Since $A_{1}+A_{2}<A_{1}^{0}+A_{2}^{0}+A_{3}^{0}=1$, it follows that

$$
\frac{\alpha A_{2}}{\alpha+1}<\frac{\alpha_{0} A_{2}^{0}}{\alpha_{0}+1}+A_{3}^{0} .
$$

From (5), we deduce that there exist $p, q \in \mathbb{N}, p>0, q>0$, such that

$$
\max \left\{\frac{\alpha_{0} A_{2}^{0} /\left(\alpha_{0}+1\right)}{\alpha \alpha_{0}\left(h_{1}+h_{2}\right)}, \frac{\alpha A_{2} /(\alpha+1)}{\alpha \alpha_{0}\left(h_{1}+h_{2}\right)}\right\}<\frac{p}{q}<\frac{\alpha_{0} A_{2}^{0} /\left(\alpha_{0}+1\right)+A_{3}^{0}}{\alpha \alpha_{0}\left(h_{1}+h_{2}\right)} .
$$


Set

$$
x=\frac{1}{p}\left(\frac{A_{2}}{h_{2}(\alpha+1)}-1\right), \quad y=\frac{1}{q}\left(\frac{\alpha A_{2}}{h_{2}(\alpha+1)}-\alpha_{0}\right), \quad z=\alpha \frac{p}{q}\left(h_{1}+h_{2}\right)-h_{2} .
$$

By the choice of $p, q$, it is straight forward to verify that $z$ satisfies (4). We have

$$
\begin{aligned}
& u_{1}=\left(x, h_{1}\right)=\left(\frac{A_{2}}{p h_{2}(\alpha+1)}-\frac{1}{p}, h_{1}\right), \\
& w_{1}=(y, z)=\left(\frac{\alpha A_{2}}{q h_{2}(\alpha+1)}-\frac{\alpha_{0}}{q}, \alpha \frac{p}{q}\left(h_{1}+h_{2}\right)-h_{2}\right) . \\
& v_{1}^{\prime}=v_{1}+p\left(u_{1}+w_{2}\right)=\left(\frac{A_{2}}{h_{2}(\alpha+1)}, p\left(h_{1}+h_{2}\right)\right) \\
& v_{2}^{\prime}=v_{2}+q\left(w_{1}+w_{2}\right)=\alpha\left(\frac{A_{2}}{h_{2}(\alpha+1)}, p\left(h_{1}+h_{2}\right)\right) .
\end{aligned}
$$

Since $\Lambda_{3}^{\vee}$ is generated by $v_{1}$ and $u_{1}+w_{2}$, we see that $v_{1}^{\prime}$ is a primitive vector in $\Lambda_{3}^{\vee}$, similarly, $v_{2}^{\prime}$ is a primitive vector in $\Lambda_{2}^{\vee}$. Clearly, we have $v_{2}^{\prime}=\alpha v_{1}^{\prime}$, hence (i) is satisfied. We have

$$
\left|v_{1}^{\prime} \wedge w_{2}\right|=\left|\begin{array}{cc}
A_{2} /\left(h_{2}(\alpha+1)\right) & 0 \\
p\left(h_{1}+h_{2}\right) & h_{2}
\end{array}\right|=\frac{A_{2}}{\alpha+1},
$$

therefore (ii) is satisfied. Next, we have

$$
\operatorname{Area}\left(T_{2}^{\vee}\right)=\left|v_{2} \wedge\left(w_{1}+w_{2}\right)\right|=\alpha \alpha_{0} \frac{p}{q}\left(h_{1}+h_{2}\right)
$$

and

$$
\left|v_{2}^{\prime} \wedge w_{2}\right|=\alpha\left|v_{1}^{\prime} \wedge w_{2}\right|=\frac{\alpha A_{2}}{\alpha+1} .
$$

By the choice of $p, q$, we have $\left|v_{2}^{\prime} \wedge w_{2}\right|<\operatorname{Area}\left(T_{2}^{\vee}\right)$, hence (iii) is satisfied.

Claim 2 Given $(x, y, z)$ as in Claim 1, there exist $\hat{u}_{1}$ satisfying (3) such that $v_{2}^{\prime}$ is generic with respect to the lattice $\mathbb{Z} w_{1} \oplus \mathbb{Z}\left(v_{2}^{\prime}+\widehat{u}_{1}\right)$.

Proof of Claim 2 Since

$$
\left|v_{2}^{\prime} \wedge w_{1}\right|=\alpha_{0}\left(h_{1}+h_{2}\right) \frac{p}{q}-\frac{A_{2}}{\alpha+1}>0,
$$

we deduce that $\left\{v_{2}^{\prime}, w_{1}\right\}$ is a basis of $\mathbb{R}^{2}$. Therefore, we can write $\hat{u}_{1}=\lambda w_{1}+\mu v_{2}^{\prime}$. Observe that, once $w_{1}$ is fixed, the set of $\hat{u}_{1}$ satisfying (3) is parameterized by $\lambda \in \mathbb{R}$, with fixed $\mu$.

Observe that $v_{2}^{\prime}$ is parallel to a vector in $\mathbb{Z} w_{1} \oplus \mathbb{Z}\left(v_{2}^{\prime}+\widehat{u}_{1}\right)$ if and only if $\lambda \in \mathbb{Q}$. Indeed, suppose that $v_{2}^{\prime}=\lambda^{\prime}\left(m w_{1}+n\left(v_{2}^{\prime}+\widehat{u}_{1}\right)\right)$, with $m, n \in \mathbb{Z}$. Then we must 
have $n \neq 0$, otherwise $v_{2}^{\prime}$ and $w_{1}$ are colinear, therefore $\hat{u}_{1}=-(m / n) w_{1}+\lambda^{\prime \prime} v_{2}^{\prime}$. It follows immediately that there exist $\hat{u}_{1}$ satisfying (3) such that $v_{2}^{\prime}$ is generic with respect to $\mathbb{Z} w_{1} \oplus \mathbb{Z}\left(v_{2}^{\prime}+\widehat{u}_{1}\right)$.

Let us now show that the lemma will follow from Claims 1 and 2. Choose $(x, y, z)$ as in Claim 1, and choose $\hat{u}_{1}$ as in Claim 2. Then the parameters $\left(x, y, z, \hat{u}_{1}\right)$ give us an element $X$ in $\operatorname{SSpl}\left(A_{1}^{0}, A_{2}^{0}, A_{3}^{0}, \alpha_{0}\right)$. We have $\Sigma=\Psi(X) \in \overline{\operatorname{SL}(2, \mathbb{R}) \cdot \Psi\left(X_{0}\right)}$.

Let $\sigma_{1}^{ \pm}$(resp. $\sigma_{2}^{ \pm}$) be the pair of saddle connections in $T_{3}^{\vee}$ (resp. in $T_{2}^{\vee}$ ) corresponding to $v_{1}^{\prime}$ (resp. $\left.v_{2}^{\prime}\right)$. Since

$$
\left|v_{1}^{\prime} \wedge w_{2}\right|=\frac{A_{2}}{\alpha+1}<\operatorname{Area}\left(T_{3}^{\vee}\right)=\frac{A_{2}^{0}}{\alpha_{0}+1}+A_{1}^{0},
$$

from Lemma 4.1, we deduce that $\sigma_{1}^{ \pm}$meet $\eta_{2}$ at only one point. Consequently, we see that $\sigma_{1}^{ \pm}$bound a simple cylinder containing $\eta_{2}$. Similarly, since $\operatorname{Area}\left(T_{2}^{\vee}\right)=$ $\left|v_{2}^{\prime} \wedge w_{1}\right|+\left|v_{2}^{\prime} \wedge w_{2}\right|$, it follows that $\sigma_{2}^{ \pm}$cut $T_{2}^{\vee}$ into two cylinders, one contains $\eta_{1}$, the other contains $\eta_{2}$. Consequently, $\sigma_{1}^{ \pm}$and $\sigma_{2}^{ \pm}$give rise to two pairs of homologous saddle connections in $\Sigma$ which determine a special splitting $X^{\prime}=\left(T_{1}^{\prime}, T_{2}^{\prime}, T_{3}^{\prime}, v_{1}^{\prime}, v_{2}^{\prime}\right)$. We have

$$
\begin{aligned}
& \operatorname{Area}\left(T_{1}^{\prime}\right)=\operatorname{Area}\left(T_{3}^{\vee}\right)-\left|v_{1}^{\prime} \wedge w_{2}\right|=\frac{A_{2}}{\alpha+1}+A_{1}-\frac{A_{2}}{\alpha+1}=A_{1} . \\
& \operatorname{Area}\left(T_{2}^{\prime}\right)=\left|\left(v_{1}^{\prime}+v_{2}^{\prime}\right) \wedge w_{2}\right|=A_{2} .
\end{aligned}
$$

Therefore, $\operatorname{Area}\left(T_{3}^{\prime}\right)=A_{3}$. Since $\Lambda\left(T_{3}^{\prime}\right)=\mathbb{Z} w_{1} \oplus \mathbb{Z}\left(v_{2}^{\prime}+\widehat{u}_{1}\right)$, it follows from the choice of $\hat{u}_{1}$ that $v_{2}^{\prime}$ is generic with respect to $\Lambda\left(T_{3}^{\prime}\right)$. We can then conclude that for any $\left(A_{1}, A_{2}, A_{3}, \alpha\right) \in \mathbb{R}_{>0}^{4}$ such that

$$
\begin{aligned}
A_{1}+A_{2}+A_{3} & =1, \\
A_{1}+\frac{A_{2}}{\alpha+1} & =A_{1}^{0}+\frac{A_{2}^{0}}{\alpha_{0}+1},
\end{aligned}
$$

there exist $X^{\prime}=\left(T_{1}^{\prime}, T_{2}^{\prime}, T_{3}^{\prime}, v_{1}^{\prime}, v_{2}^{\prime}\right) \in \operatorname{SSpl}\left(A_{1}, A_{2}, A_{3}, \alpha\right)$, with $v_{2}^{\prime}$ generic with respect to $\Lambda\left(T_{3}^{\prime}\right)$, such that $\Psi\left(X^{\prime}\right) \in \operatorname{SL}(2, \mathbb{R}) \cdot \Psi\left(X_{0}\right)$. We can now complete the proof of Lemma 5.2 as follows: first, for any $\left(A_{1}, A_{2}, A_{3}, \alpha\right)$ such that $\left(A_{1} / A_{2}\right)(\alpha+1)^{2} \notin \mathbb{Q}$, it follows from Lemma 4.6 that $\Psi\left(\operatorname{SSpl}\left(A_{1}, A_{2}, A_{3}, \alpha\right)\right) \subset \overline{\operatorname{SL}(2, \mathbb{R}) \cdot \Psi\left(X_{0}\right)}$. By continuity of $\Psi$, it follows that $\overline{\operatorname{SL}(2, \mathbb{R}) \cdot \Psi\left(X_{0}\right)}$ contains $\Psi\left(\operatorname{SSpl}\left(A_{1}, A_{2}, A_{3}, \alpha\right)\right)$ for all $\left(A_{1}, A_{2}, A_{3}, \alpha\right)$. 
To complete the proof of Proposition 5.1, we need the following:

Lemma 5.3 For any $\left(A_{1}, A_{2}, A_{3}, \alpha\right)$ such that

$$
\begin{aligned}
& A_{1}+A_{2}+A_{3}=1, \\
& A_{1}+\frac{A_{2}}{\alpha+1}<1-\left(A_{1}^{0}+\frac{A_{2}^{0}}{\alpha_{0}+1}\right)=\frac{\alpha_{0} A_{2}^{0}}{\alpha_{0}+1}+A_{3}^{0},
\end{aligned}
$$

we have $\Psi\left(\operatorname{SSpl}\left(A_{1}, A_{2}, A_{3}, \alpha\right)\right) \subset \overline{\operatorname{SL}(2, \mathbb{R}) \cdot \Psi\left(X_{0}\right)}$.

Proof Since

$$
A_{1}+\frac{A_{2}}{\alpha+1}<1-\left(A_{1}^{0}+\frac{A_{2}^{0}}{\alpha_{0}+1}\right),
$$

we can find $\left(A_{1}^{\prime}, A_{2}^{\prime}, A_{3}^{\prime}, \alpha^{\prime}\right) \in \mathbb{R}_{>0}^{4}$ such that

$$
\begin{aligned}
A_{1}^{\prime}+A_{2}^{\prime}+A_{3}^{\prime} & =1, \\
A_{1}^{\prime}+\frac{A_{2}^{\prime}}{\alpha^{\prime}+1} & =A_{1}^{0}+\frac{A_{2}^{0}}{\alpha_{0}+1}, \\
A_{3}^{\prime} & =A_{1}+\frac{A_{2}}{\alpha+1} .
\end{aligned}
$$

From Lemma 5.2, we know that $\Psi\left(\operatorname{SSpl}\left(A_{1}^{\prime}, A_{2}^{\prime}, A_{3}^{\prime}, \alpha^{\prime}\right)\right) \subset \overline{\operatorname{SL}(2, \mathbb{R}) \cdot \Psi\left(X_{0}\right)}$. Consider an element $X=\left(T_{1}, T_{2}, T_{3}, v_{1}, v_{2}\right) \in \operatorname{SSpl}\left(A_{1}^{\prime}, A_{2}^{\prime}, A_{3}^{\prime}, \alpha^{\prime}\right)$, where $v_{1}=(1,0)$, $v_{2}=\left(\alpha^{\prime}, 0\right)$. Let $\Lambda_{i}$ denote the lattice associated to $T_{i}, i=1,2,3$. Observe that we can choose $X$ such that (see Figure 8)

- $\Lambda_{1}$ contains no vertical vectors,

- $\Lambda_{2}$ contains a vector vertical vector $w_{2}$ such that $\Lambda_{2}=\mathbb{Z}\left(v_{1}+v_{2}\right) \oplus \mathbb{Z} w_{2}$,

- $\Lambda_{3}=\mathbb{Z} v_{3} \oplus \mathbb{Z} w_{1}$, where $v_{3}$ is horizontal, and $w_{1}$ is vertical.

By assumption, we see that all $\Lambda_{1}, \Lambda_{2}, \Lambda_{3}$ contain horizontal vectors. Let $C_{i}, i=$ $1,2,3$, denote the horizontal cylinder obtained by slitting $T_{i}$ along the horizontal saddle connections, which correspond to the primitive horizontal vectors in $T_{i}$. Let $\ell_{i}$ and $h_{i}$ denote width and the height of $C_{i}$. Note that $h_{1}, h_{2}$ are determined by $\left(A_{1}^{\prime}, A_{2}^{\prime}, A_{3}^{\prime}, \alpha^{\prime}\right)$, and $\ell_{3}$ and $h_{3}$ must satisfy $\ell_{3}>\alpha^{\prime}$ and $\ell_{3} h_{3}=A_{3}^{\prime}$.

By construction, the surface $\Sigma$ constructed from $X$ admits another special splitting $X^{\vee}=\left(T_{1}^{\vee}, T_{2}^{\vee}, T_{3}^{\vee}, w_{1}, w_{2}\right)$ which is dual to $X$. Since $\Lambda_{1}$ contains no vertical vectors, the lattice $\Lambda_{3}^{\vee}$ does not contain any vertical vector. Let $\bar{m}^{\vee}$ denote the moduli ratio of $X^{\vee}$ :

$$
\bar{m}^{\vee}=\frac{\left(\ell_{3}-\alpha^{\prime}\right)\left(h_{2}+h_{3}\right)}{\alpha^{\prime} h_{2}} .
$$




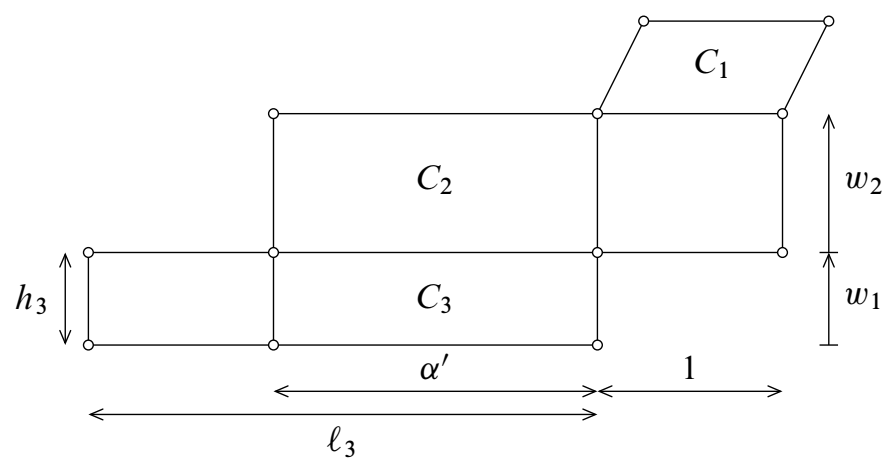

Figure 8: Switching between horizontal and vertical splittings

Since $\ell_{3}=A_{3}^{\prime} / h_{3}$, we see that $\bar{m}^{\vee}$ is a nonconstant rational function of $h_{3}$. Therefore, we can find $h_{3}$ so that $\bar{m}^{\vee} \notin \mathbb{Q}$. We deduce that there exists an element $X$ in $\operatorname{SSpl}\left(A_{1}^{\prime}, A_{2}^{\prime}, A_{3}^{\prime}, \alpha^{\prime}\right)$ such that the element $X^{\vee}$ defined above satisfies the conditions of Theorem 4.3. Let $A_{i}^{\vee}, i=1,2,3$, denote the area of $T_{i}^{\vee}$, and $\alpha^{\vee}=\left|w_{2}\right| /\left|w_{1}\right|$. By construction, we have

$$
A_{1}^{\vee}+\frac{A_{2}^{\vee}}{\alpha^{\vee}+1}=A_{3}^{\prime}=A_{1}+\frac{A_{2}}{\alpha+1} .
$$

Therefore, it follows from Lemma 5.2 that

$$
\Psi\left(\operatorname{SSpl}\left(A_{1}, A_{2}, A_{3}, \alpha\right)\right) \subset \overline{\operatorname{SL}(2, R) \cdot \Psi\left(X^{\vee}\right)} \subset \overline{\operatorname{SL}(2, \mathbb{R}) \cdot \Psi\left(X_{0}\right)} .
$$

\subsection{Proof of Proposition 5.1}

All we need to show is that $\Psi\left(\operatorname{SSpl}\left(A_{1}, A_{2}, A_{3}, \alpha\right)\right) \subset \overline{\operatorname{SL}(2, \mathbb{R}) \cdot \Psi\left(X_{0}\right)}$ for all $\left(A_{1}, A_{2}, A_{3}, \alpha\right)$ such that $A_{1}+A_{2}+A_{3}=1$. Choose $\left(A_{1}^{\prime}, A_{2}^{\prime}, A_{3}^{\prime}, \alpha^{\prime}\right)$ in $\mathbb{R}_{>0}^{4}$ so that

$$
A_{1}^{\prime}+\frac{A_{2}^{\prime}}{\alpha^{\prime}+1}<\min \left\{1-\left(A_{1}^{0}+\frac{A_{2}^{0}}{\alpha_{0}+1}\right), 1-\left(A_{1}+\frac{A_{2}}{\alpha+1}\right)\right\} .
$$

By Lemma 5.3, we know that $\Psi\left(\operatorname{SSpl}\left(A_{1}^{\prime}, A_{2}^{\prime}, A_{3}^{\prime}, \alpha^{\prime}\right)\right) \subset \overline{\operatorname{SL}(2, \mathbb{R}) \cdot \Psi\left(X_{0}\right)}$. Let $X$ be an element in $\operatorname{SSpl}\left(A_{1}^{\prime}, A_{2}^{\prime}, A_{3}^{\prime}, \alpha^{\prime}\right)$ which satisfies the conditions of Theorem 4.3. Since we have

$$
A_{1}+\frac{A_{2}}{\alpha+1}<1-\left(A_{1}^{\prime}+\frac{A_{2}^{\prime}}{\alpha^{\prime}+1}\right),
$$

by applying Lemma 5.3 with $X$ in the place of $X_{0}$, we see that

$$
\Psi\left(\operatorname{SSpl}\left(A_{1}, A_{2}, A_{3}, \alpha\right)\right) \subset \overline{\operatorname{SL}(2, \mathbb{R}) \cdot \Psi(X)} \subset \overline{\operatorname{SL}(2, \mathbb{R}) \cdot \Psi\left(X_{0}\right)},
$$

and the proposition follows. 


\section{Proof of Theorem 4.3}

By Proposition 5.1, we know that $\overline{\mathcal{O}}=\overline{\operatorname{SL}(2, \mathbb{R}) \cdot \Psi\left(X_{0}\right)}$ contains all the surfaces that admit a special splitting. We will show that $\overline{\mathcal{O}}$ contains all the Veech surfaces in $\mathcal{H}_{1}^{\text {hyp }}(4)$, in particular, $\overline{\mathcal{O}}$ contains all the square-tiled surfaces. Since the set of square-tiled surfaces is dense in $\mathcal{H}_{1}^{\text {hyp }}(4)$ (see [9, Lemma 4]), it follows immediately that $\overline{\mathcal{O}}=\mathcal{H}_{1}^{\text {hyp }}(4)$.

Let $\Sigma$ be a Veech surface in $\mathcal{H}_{1}^{\text {hyp }}(4)$. From Corollary 3.2, we know that there exists on $\Sigma$ a pair of homologous saddle connections $\delta^{ \pm}$such that by cutting along $\delta^{ \pm}$, and gluing the two geodesic segments in the boundary of each of the connected component obtained from the cutting, we get a surface in $\mathcal{H}(0,0)$, which will be denoted by $\Sigma^{\prime}$, and a surface in $\mathcal{H}(2)$ which will be denoted by $\Sigma^{\prime \prime}$. On both $\Sigma^{\prime}$ and $\Sigma^{\prime \prime}$ we have a marked saddle connection corresponding to the pair $\delta^{ \pm}$, we denote both of them by $\delta$. Without loss of generality, we can assume that $\delta$ is horizontal. Since $\Sigma$ is a Veech surface, the Veech dichotomy (see [8;11]) implies that $\Sigma$ is decomposed into cylinders which are filled with horizontal closed geodesics. In particular, we see that $\Sigma^{\prime \prime}$ is a union of horizontal cylinders. We have two possibilities:

Case $1 \Sigma^{\prime \prime}$ is the union of two cylinders. In this case, there exists another pair of homologous horizontal saddle connections $\gamma^{ \pm}$in $\Sigma^{\prime \prime}$ which, together with $\delta^{ \pm}$, determine a special splitting of $\Sigma$. Therefore, $\Sigma \in \overline{\mathcal{O}}$ by Proposition 5.1.

Case $2 \Sigma^{\prime \prime}$ contains only one horizontal cylinder. In this case, there exist two other horizontal saddle connections $\gamma_{1}, \gamma_{2}$ in $\Sigma$ such that $\delta * \gamma_{1} * \gamma_{2}$ is freely homotopic to a simple closed geodesic. Consequently, $\Sigma^{\prime \prime}$ can be constructed from a single parallelogram $\mathrm{P}$ by the gluing as shown in Figure 9. Actually, $\mathrm{P}$ is an octagon whose opposite sides are parallel and have the same length. Let $U=(x, 0), V_{1}=(y, 0), V_{2}=$ $(z, 0)$ with $x>0, y>0, z>0$ be the vectors associated to the saddle connections $\delta, \gamma_{1}, \gamma_{2}$ respectively.
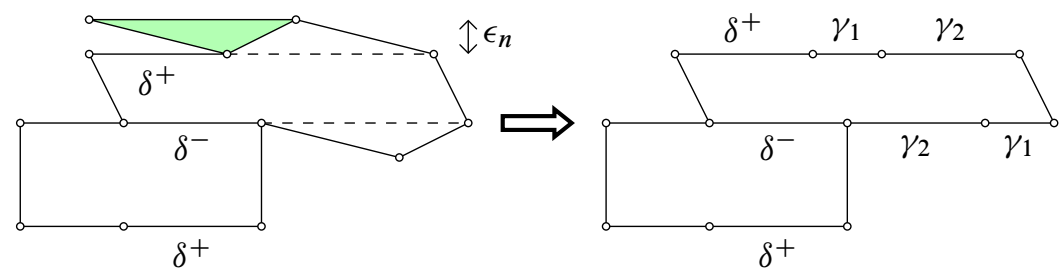

Figure 9: Surfaces with special splitting converging to $\Sigma$

Let $\left\{\epsilon_{n}\right\}$ be a sequence of positive real number decreasing to zero. For each $\epsilon_{n}$, we construct a surface $\Sigma_{n}$ in $\mathcal{H}_{1}^{\text {hyp }}(4)$ as follows: first, we construct a surface $\Sigma_{n}^{\prime \prime}$ from an 
octagon $\mathrm{P}_{n}$, which is obtained from $\mathrm{P}$ by replacing $V_{1}$ by the vector $V_{1}^{(n)}=\left(y, \epsilon_{n}\right)$, and $V_{2}$ by the vector $V_{2}^{(n)}=\left(z,-\epsilon_{n}\right)$. Then we glue $\Sigma_{n}^{\prime \prime}$ to $\Sigma^{\prime}$ along the pair of homologous saddle connections $\delta^{ \pm}$, and rescale to get a surface in $\mathcal{H}_{1}^{\text {hyp }}(4)$. By construction, we see that $\Sigma_{n}$ admits a special splitting by horizontal saddle connections, therefore $\Sigma_{n} \in \overline{\mathcal{O}}$. As $\epsilon_{n} \longrightarrow 0$, the sequence $\left\{\Sigma_{n}\right\}$ converges to $\Sigma$, hence we have $\Sigma \in \overline{\mathcal{O}}$.

The proof of Theorem 4.3 is now complete.

\section{Proof of Theorem 1.2}

We can now prove Theorem 1.2 as a consequence of Theorem 4.3. The idea is to show that there exists in the closure of $\operatorname{SL}(2, \mathbb{R}) \cdot \Psi\left(X_{0}\right)$ a surface which admits a special splitting satisfying the conditions of Theorem 4.3. As usual, let $A_{i}^{0}$ and $\Lambda_{i}^{0}$ denote the area and the associated lattice of $T_{i}^{0}, i=1,2,3$. We can assume that $v_{1}^{0}=(1,0)$ and $v_{2}^{0}=\left(\alpha_{0}, 0\right)$. Let $t_{1}^{0}$ and $t_{2}^{0}$ denote the twists of the pairs $\left(T_{1}^{0}, v_{1}^{0}\right)$ and $\left(T_{2}^{0}, v_{1}^{0}+v_{2}^{0}\right)$ respectively (see 4.3). Obviously, we only have to consider the case

$$
\bar{m}_{0}=m_{1}^{0} / m_{2}^{0}=\frac{A_{1}^{0}}{A_{2}^{0}}\left(\alpha_{0}+1\right)^{2} \in \mathbb{Q} .
$$

Let $n_{1}, n_{2}$ be the integers such that $\operatorname{gcd}\left(n_{1}, n_{2}\right)=1$ and $n_{1} m_{1}^{0}+n_{2} m_{2}^{0}=0$. Ratner's Theorem for the action of

$$
U=\left\{\left(\begin{array}{ll}
1 & t \\
0 & 1
\end{array}\right), t \in \mathbb{R}\right\}
$$

implies $\overline{U \cdot \Psi\left(X_{0}\right)} \supset \Psi(X)$ for all $X=\left(T_{1}, T_{2}, T_{3}, v_{1}, v_{2}\right) \in \operatorname{SSpl}\left(A_{1}^{0}, A_{2}^{0}, A_{3}^{0}, \alpha_{0}\right)_{\text {hor }}$ such that

$$
n_{1}\left(t_{1}-t_{1}^{0}\right)+n_{2}\left(t_{2}-t_{2}^{0}\right) \in \mathbb{Z},
$$

where $t_{1}, t_{2}$ are the twists of the pairs $\left(T_{1}, v_{1}\right)$ and $\left(T_{2}, v_{1}+v_{2}\right)$ respectively. Consider such an $X$ with $t_{2}=0$. Let $\Lambda_{i}$ denote the lattice associated to $T_{i}, i=1,2,3$. Since $t_{2}=0$, the lattice $\Lambda_{2}$ contains vertical vectors, let $w_{1}$ be the primitive vertical vector in $\Lambda_{2}$, and let $\eta_{2}^{ \pm}$denote the pair of homologous saddle connections in $T_{2}$ such that $V\left(\eta_{2}^{ \pm}\right)=w_{2}$. Let $w_{1}$ be a primitive vector in the lattice $\Lambda_{3}$ such that $\left|w_{1} \wedge v_{2}^{0}\right|<A_{3}^{0}$, and let $\eta_{1}^{ \pm}$denote the pair of homologous saddle connections in $T_{3}$ such that $V\left(\eta_{1}^{ \pm}\right)=w_{1}$. The saddle connections $\eta_{1}^{ \pm}$and $\eta_{2}^{ \pm}$determine a splitting $X^{\vee}=\left(T_{1}^{\vee}, T_{2}^{\vee}, T_{3}^{\vee}, w_{1}, w_{2}\right)$ of the surface $\Sigma=\Psi(X)$. Let $\Lambda_{i}^{\vee}$ and $A_{i}^{\vee}$ denote the associated lattice and the area of $T_{i}^{\vee}, i=1,2,3$. Here we have two cases: 


\section{Case $1 \quad t_{1} \notin \mathbb{Q}$.}

In this case, the lattice $\Lambda_{3}^{\vee}$ does not contain any vertical vector. We can choose $w_{1}$ to be vertical and $A_{1}^{\vee} / A_{2}^{\vee}\left(\alpha^{\vee}+1\right)^{2} \notin \mathbb{Q}$, where $\alpha^{\vee}=\left|w_{2}\right| /\left|w_{1}\right|$ (see Lemma 5.3). Hence the splitting $X^{\vee}$ satisfies the conditions of Theorem 4.3, it follows immediately that $\overline{\operatorname{SL}(2, \mathbb{R}) \cdot \Psi\left(X_{0}\right)}=\overline{\operatorname{SL}(2, \mathbb{R}) \cdot \Psi\left(X^{\vee}\right)}=\mathcal{H}_{1}^{\text {hyp }}(4)$.

Case $2 t_{1} \in \mathbb{Q}$.

In this case, $\Lambda_{3}^{\vee}$ contains vertical vectors. Let $\hat{u}_{3}=\left(0, h_{3}\right)$, with $h_{3}>0$, be the primitive vertical vector of $\Lambda_{3}^{\vee}$, and $\hat{v}_{3}=(\ell, h)$, with $\ell>0$ and $0 \leqslant h<h_{3}$, be another primitive vector such that $\Lambda_{3}^{\vee}=\mathbb{Z} \hat{u}_{3} \oplus \mathbb{Z} \hat{v}_{3}$.

By assumption, we have $w_{2}=\left(0, h_{2}\right)$, with $h_{2}>0$. Remark that we must have $h_{3}>h_{2}$. Recall that we are free to choose $T_{3}$ and $w_{1}$ provided $\operatorname{Area}\left(T_{3}\right)=A_{3}^{0}$, and $\left|w_{1} \wedge v_{2}^{0}\right|<A_{3}^{0}$. By construction, we have $\Lambda_{2}^{\vee}=\mathbb{Z} v_{2}^{0} \oplus \mathbb{Z}\left(w_{1}+w_{2}\right)$. The theorem follows from the following observation (see also Lemma 5.2)

Claim We can choose $\Lambda_{3}$ and $w_{1}$ so that there exist a primitive vector $v_{1}^{\prime}$ of $\Lambda_{3}^{\vee}$, and a primitive vector $v_{2}^{\prime}$ of $\Lambda_{2}^{\vee}$ such that the surface $\Sigma$ admits a special splitting $X^{\prime}=\left(T_{1}^{\prime}, T_{2}^{\prime}, T_{3}^{\prime}, v_{1}^{\prime}, v_{2}^{\prime}\right)$ dual to $X^{\vee}$ which satisfies the conditions of Theorem 4.3.

Proof of Claim Set $A_{1}=\ell\left(h_{3}-h_{2}\right)>0$, and choose $\left(A_{2}, A_{3}, \alpha\right)$ in $\mathbb{R}_{>0}^{3}$ such that

$$
\begin{aligned}
& A_{1}+A_{2}+A_{3}=1, \\
& A_{1}+\frac{A_{2}}{\alpha+1}=A_{1}^{0}+\frac{A_{2}^{0}}{\alpha_{0}+1}, \\
& \frac{A_{1}}{A_{2}}(\alpha+1)^{2} \notin \mathbb{Q} .
\end{aligned}
$$

Since

$$
A_{1}+\frac{A_{2}}{\alpha+1}=A_{1}^{0}+\frac{A_{2}^{0}}{\alpha_{0}+1}=A_{3}^{\vee}=\ell h_{3}
$$

and $A_{1}=\ell\left(h_{3}-h_{2}\right)$, it follows that $A_{2} /(\alpha+1)=\ell h_{2}$. Thus

$$
\frac{\alpha A_{2}}{\alpha+1}=\alpha \ell h_{2}<1-\left(A_{1}^{0}+\frac{A_{2}^{0}}{\alpha_{0}+1}\right)=\frac{\alpha_{0} A_{2}^{0}}{\alpha_{0}+1}+A_{3}^{0}=: A_{0} \text {. }
$$

Choose $q \in \mathbb{N}$ large enough so that

$$
\left\{\begin{array}{l}
\frac{h}{h_{3} q}<\frac{\alpha \ell h_{2}}{\alpha \alpha_{0} h_{3}}, \\
\frac{1}{q}<\frac{1}{2} \frac{A_{0}-\alpha \ell h_{2}}{\alpha \alpha_{0} h_{3}} .
\end{array}\right.
$$




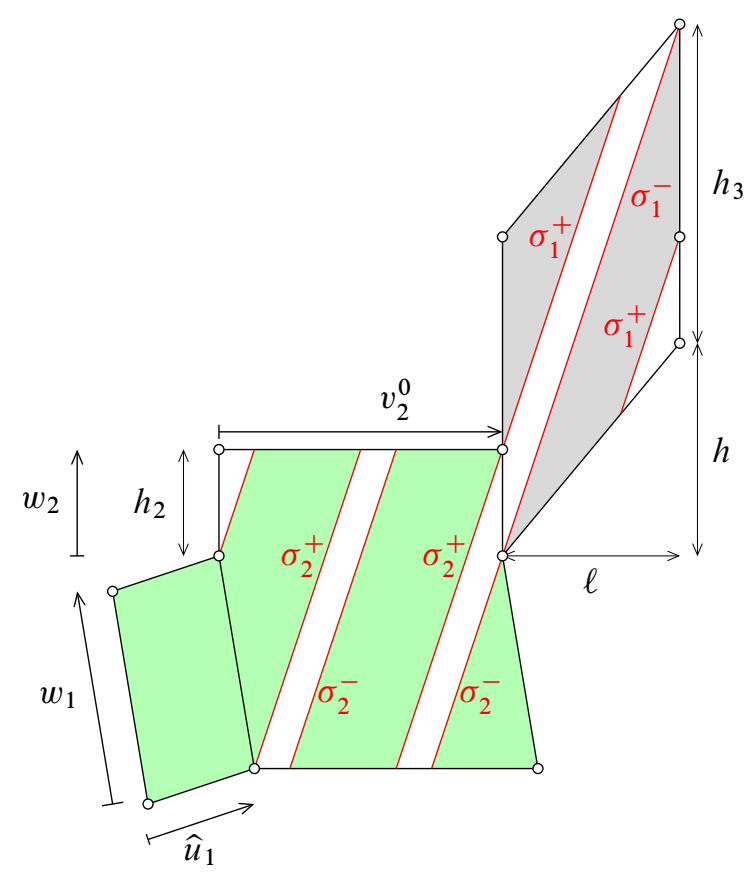

Figure 10: Case $t_{1} \in \mathbb{Q}$, ie the lattice $\Lambda_{3}^{\vee}$ contains vertical vectors

From (6), it follows that there exists $p \in \mathbb{N}$ such that

Now, we can take

$$
\frac{\alpha \ell h_{2}}{\alpha \alpha_{0} h_{3}}-\frac{h}{h_{3} q}<\frac{p}{q}<\frac{A_{0}}{\alpha \alpha_{0} h_{3}}-\frac{h}{h_{3} q} \text {. }
$$

$$
\begin{aligned}
v_{1}^{\prime} & =p \hat{u}_{3}+\widehat{v}_{3}=\left(\ell, p h_{3}+h\right), \\
w_{1} & =\left(\frac{\alpha \ell-\alpha_{0}}{q}, \alpha h_{3}\left(\frac{h}{h_{3} q}+\frac{p}{q}\right)-h_{2}\right), \\
v_{2}^{\prime} & =v_{2}^{0}+q\left(w_{1}+w_{2}\right)=\left(\alpha \ell, \alpha\left(p h_{3}+h\right)\right) .
\end{aligned}
$$

Observe that $v_{1}^{\prime}$ and $v_{2}^{\prime}$ are primitive vectors in $\Lambda_{3}^{\vee}$ and $\Lambda_{2}^{\vee}$ respectively. Clearly, we have $v_{2}^{\prime}=\alpha v_{1}^{\prime}$. By the choice of $p, q$, we also have

$$
\begin{aligned}
\left|v_{1}^{\prime} \wedge w_{2}\right| & =\ell h_{2}<\ell h_{3}=A_{3}^{\vee}=A_{1}^{0}+\frac{A_{2}^{0}}{\alpha_{0}+1}, \\
\left|v_{2}^{\prime} \wedge w_{2}\right| & =\alpha \ell h_{2}<\alpha_{0} \alpha h_{3}\left(\frac{h}{h_{3} q}+\frac{p}{q}\right)=\left|v_{2}^{0} \wedge\left(w_{1}+w_{2}\right)\right|=A_{2}^{\vee}, \\
A_{2}^{\vee} & =\alpha_{0} \alpha h_{3}\left(\frac{h}{h_{3} q}+\frac{p}{q}\right)<A_{0}=1-A_{3}^{\vee} .
\end{aligned}
$$


Consequently, the surface $\Sigma$ admits a special splitting determined by two pairs of homologous saddle connections $\sigma_{1}^{ \pm}$and $\sigma_{2}^{ \pm}$, where $\sigma_{1}^{ \pm}$is the pair of saddle connections in $T_{3}^{\vee}$ corresponding to $v_{1}^{\prime}$, and $\sigma_{2}^{ \pm}$is the pair of saddle connections in $T_{2}^{\vee}$ corresponding to $v_{2}^{\prime}$ (see Figure 10). Let $X^{\prime}=\left(T_{1}^{\prime}, T_{2}^{\prime}, T_{3}^{\prime}, v_{1}^{\prime}, v_{2}^{\prime}\right)$ denote this special splitting. Then we have $\operatorname{Area}\left(T_{i}^{\prime}\right)=A_{i}, i=1,2,3$.

By construction, $w_{1}$ is a primitive vector of $\Lambda_{1}^{\vee}$. Let $\hat{u}_{1}$ be another primitive such that $\Lambda_{1}^{\vee}=\mathbb{Z} w_{1} \oplus \mathbb{Z} \hat{u}_{1}$. Recall that we can choose $T_{3}$ arbitrarily provided $\operatorname{Area}\left(T_{3}\right)=A_{3}^{0}$, therefore we are free to choose $\widehat{u}_{1}$, provided $\left|\widehat{u}_{1} \wedge w_{1}\right|=A_{1}^{\vee}=1-\left(A_{2}^{\vee}+A_{3}^{\vee}\right)$. It is easy to check that we can choose such a $\widehat{u}_{1}$ so that the vector $v_{2}^{\prime}$ is generic with respect to $\Lambda\left(T_{3}^{\prime}\right)=\mathbb{Z} w_{1} \oplus \mathbb{Z}\left(v_{2}^{\prime}+\widehat{u}_{1}\right)$. With this choice, we see that splitting $X^{\prime}$ satisfies the conditions of Theorem 4.3 , and the claim is then proved.

By Theorem 4.3, we know that $\overline{\operatorname{SL}(2, \mathbb{R}) \cdot \Sigma}=\overline{\operatorname{SL}(2, \mathbb{R}) \cdot \Psi\left(X^{\prime}\right)}=\mathcal{H}_{1}^{\text {hyp }}(4)$. Since $\Sigma \in \overline{\operatorname{SL}(2, \mathbb{R}) \cdot \Sigma_{0}}$, it follows $\overline{\operatorname{SL}(2, \mathbb{R}) \cdot \Sigma_{0}}=\mathcal{H}_{1}^{\text {hyp }}(4)$, and Theorem 1.2 is proven.

\section{Surfaces admitting completely periodic directions with three cylinders}

\subsection{Two models of decomposition into three cylinders}

Lemma 8.1 Let $\Sigma$ be a surface in $\mathcal{H}^{\text {hyp }}(4)$. Assume that $\Sigma$ is decomposed into three horizontal cylinders, that is, the horizontal direction is completely periodic for $\Sigma$ with three cylinders. Then the surface $\Sigma$ can be reconstructed from three (horizontal) cylinders by one of the gluing models in Figure 11.

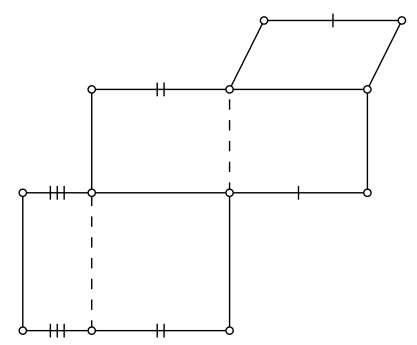

Case I

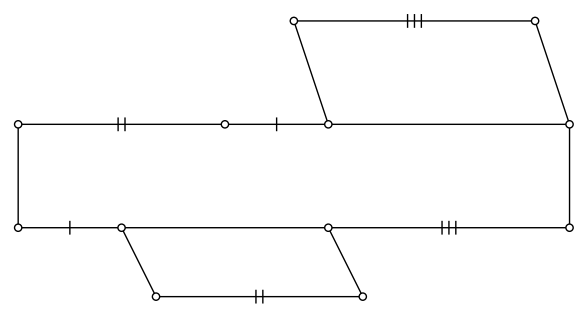

Case II

Figure 11: Two models of constructing surfaces in $\mathcal{H}^{\text {hyp }}(4)$ from three cylinders

Proof First, observe that $\Sigma$ has exactly 5 horizontal saddle connections, since the angle at the unique singular point of $\Sigma$ is $10 \pi$. Let $C_{1}, C_{2}, C_{3}$ denote the three 
horizontal cylinders. Since each of the horizontal saddle connections is contained in the lower boundary component of a unique cylinder, we then have a partition of set of horizontal saddle connections into three subsets, there are only two such partitions corresponding to two ways of writing 5 as the sum of three positive integers: $5=1+1+3=1+2+2$.

Next, let us show that the hyperelliptic involution $\tau$ of $\Sigma$ preserves each of the cylinders $C_{i}, i=1,2,3$. Consider a simple closed geodesic $c_{i}$ in $C_{i}$ close to its lower boundary. Since $\tau\left(c_{i}\right)+c_{i}=0$ in $H_{1}(\Sigma, \mathbb{Z})$, we deduce that $c_{i}$ and $\tau\left(c_{i}\right)$ cut $\Sigma$ into two connected components, each of which is equipped with a flat metric with geodesic boundaries. Since $\Sigma$ has only one singularity, one of the two components has no singularities in the interior, and must be a cylinder. Therefore, $c_{i}$ and $\tau\left(c_{i}\right)$ are contained in the same cylinder $C_{i}$. As a consequence, we see that $\tau$ maps the lower boundary of each cylinder to its upper boundary. In particular, the upper boundary and the lower boundary of each cylinder contain the same number of saddles connections, and moreover, each saddle connection in the lower boundary is paired up with a saddle connection in the upper boundary, which is its image under $\tau$.

From these two observations, it is now easy to check that there are only two ways to construct $\Sigma$ from three cylinders, which are shown in Figure 11.

Remark The fact that the hyperelliptic involution preserves each of the cylinders is already known to Kontsevich and Zorich [9, Lemma 8].

\subsection{Proof of Corollary 1.3}

8.2.1 Proof of Corollary 1.3, Case (I) In this case, let $C_{1}^{0}$ denote the unique simple cylinder of the decomposition, $C_{2}^{0}$ denote the cylinder adjacent to $C_{1}^{0}$, and $C_{3}^{0}$ the remaining cylinder. Let $\ell_{i}^{0}, h_{i}^{0}, m_{i}^{0}$ denote respectively the width, the height, and the modulus of $C_{i}^{0}$. Since $m_{1}^{0}, m_{2}^{0}, m_{3}^{0}$ are independent over $\mathbb{Q}$, by applying Ratner's Theorem for the action of $U$, we deduce that $\overline{U \cdot \Sigma_{0}}$ contains all the surfaces $\Sigma$ which are constructed from 3 horizontal cylinders $C_{1}, C_{2}, C_{3}$ by the same gluing model, whenever $C_{i}$ has the same width and height as $C_{i}^{0}$.

On each boundary component of $C_{1}$ we have a marked point which corresponds to the unique singularity of $\Sigma$. Let $v_{1}=\left(v_{1}^{x}, v_{1}^{y}\right)$ be the associated vector of any geodesic segment joining the marked point in the lower boundary to the marked point in the upper boundary. We then define the twist $t_{1}$ of $C_{1}$ to be $v_{1}^{x} / \ell_{1}^{0} \bmod \mathbb{Z}$. On each boundary component of $C_{2}$ (resp. $C_{3}$ ), we have two marked points, therefore each boundary component is the union of two geodesic segments. From Lemma 8.1, we see that each segment in the upper boundary of $C_{2}$ is paired up with a segment in the 
lower boundary component by the hyperelliptic involution. Consider such a pair of segments. Let $v_{2}=\left(v_{2}^{x}, v_{2}^{y}\right)$ be the vector associated to a segment joining the two left endpoints, we then define the twist $t_{2}$ of $C_{2}$ to be $t_{2}=v_{2}^{x} / \ell_{2}^{0} \bmod \mathbb{Z}$. The twist $t_{3}$ of $C_{3}$ is defined in the same manner.

Observe that any value of $\left(t_{1}, t_{2}, t_{3}\right)$ gives us a unique surface $\Sigma$ in $\overline{U \cdot \Sigma_{0}}$. Consider the case $t_{2}=t_{3}=0$, in that case $\Sigma$ admits a special splitting by two pairs of vertical homologous saddle connections. It is easy to see that if $t_{1}$ is not in $\mathbb{Q}$ then this splitting satisfies the condition of Theorem 1.2 , tt follows immediately that $\overline{\operatorname{SL}(2, \mathbb{R}) \cdot \Sigma_{0}}=$ $\overline{\operatorname{SL}(2, \mathbb{R}) \cdot \Sigma}=\mathcal{H}_{1}^{\text {hyp }}(4)$.

8.2.2 Proof of Corollary 1.3 Case (II) In this case, we have two simple cylinders, which will be denoted by $C_{1}^{0}$ and $C_{2}^{0}$, the remaining cylinder has 3 saddle connections in each boundary component, and will be denoted by $C_{3}^{0}$. Let $\gamma_{1}^{+}, \gamma_{2}^{+}, \gamma_{3}^{+}$denote the saddle connections contained in the upper boundary of $C_{3}^{0}$ such that $\gamma_{1}^{+}$(resp. $\gamma_{2}^{+}$) is also the lower boundary of $C_{1}^{0}$ (resp. $C_{2}^{0}$ ). Let $\gamma_{i}^{-}$denote the image of $\gamma_{i}^{+}$under $\tau$. Note that the lower boundary of $C_{3}^{0}$ is the union of $\gamma_{1}^{-}, \gamma_{2}^{-}, \gamma_{3}^{-}$, and in fact $\gamma_{3}^{+}=\gamma_{3}^{-}$ (see Figure 12).

Let $\ell_{i}, h_{i}$ denote respectively the width and the height of $C_{i}^{0}, i=1,2,3$. Since the cylinders $C_{1}^{0}$ and $C_{2}^{0}$ are simple, we define their twists $t_{1}^{0}, t_{2}^{0}$ as in Case (I). Let $\delta^{+}$ (resp. $\delta^{-}$) denote a pair of homologous saddle connections in $C_{3}^{0}$ which joins the left (resp. right) endpoints of $\gamma_{1}^{-}$and $\gamma_{1}^{+}$. Using the action of $U$, we can assume that $\delta^{ \pm}$ are vertical.

Applying the Ratner's Theorem, we see that $\overline{U \cdot \Sigma_{0}}$ contains all surfaces obtained from three cylinders $\left(C_{1}, C_{2}, C_{3}\right)$ by the same gluing model as $\left(C_{1}^{0}, C_{2}^{0}, C_{3}^{0}\right)$, provided $C_{i}$ has the same width and height as $C_{i}^{0}$. In particular, $\overline{U \cdot \Sigma_{0}}$ contains all surfaces constructed from three cylinders $\left(C_{1}, C_{2}, C_{3}\right)$ with $C_{3}=C_{3}^{0}$, and, for $i=1,2$, the twist $t_{i}$ of $C_{i}$ can be chosen arbitrarily. Let $\Sigma$ be such a surface. Cut $\Sigma$ along $\delta^{ \pm}$, then glue the geodesic segments corresponding to $\delta^{ \pm}$on each component together, we get a surface in $\mathcal{H}(0,0)$ containing $C_{1}$, which will be denoted by $\Sigma^{\prime}$, and a surface in $\mathcal{H}(2)$ containing $C_{2}$, which will be denoted by $\Sigma^{\prime \prime}$. In both of $\Sigma^{\prime}$ and $\Sigma^{\prime \prime}$, we have a marked saddle connection corresponding to $\delta^{ \pm}$, we denote both of them by $\delta$.

In $\Sigma^{\prime}$, for any $\left.t_{1} \in\right] 0,1\left[\right.$, we have a pair of homologous saddle connections $\sigma_{1}^{ \pm}$such that $V\left(\sigma_{1}^{ \pm}\right)=w_{1}=\left(t_{1} \ell_{1}, h_{1}+h_{3}\right)$. This pair of saddle connections cut $\Sigma^{\prime}$ into two cylinders, one of which contains $\delta$.

Suppose that $\left.t_{2} \in\right] 0,1\left[\right.$. Then there exists a pair of homologous saddle connections $\sigma_{2}^{ \pm}$ in $\Sigma^{\prime \prime}$ such that $V\left(\sigma_{2}^{ \pm}\right)=w_{2}=\left(t_{2} \ell_{2}, h_{2}+h_{3}\right)$, which bound a simple cylinder containing $\delta$. If we cut off the simple cylinder bounded by $\sigma_{2}^{ \pm}$from $\Sigma^{\prime \prime}$, and then glue 


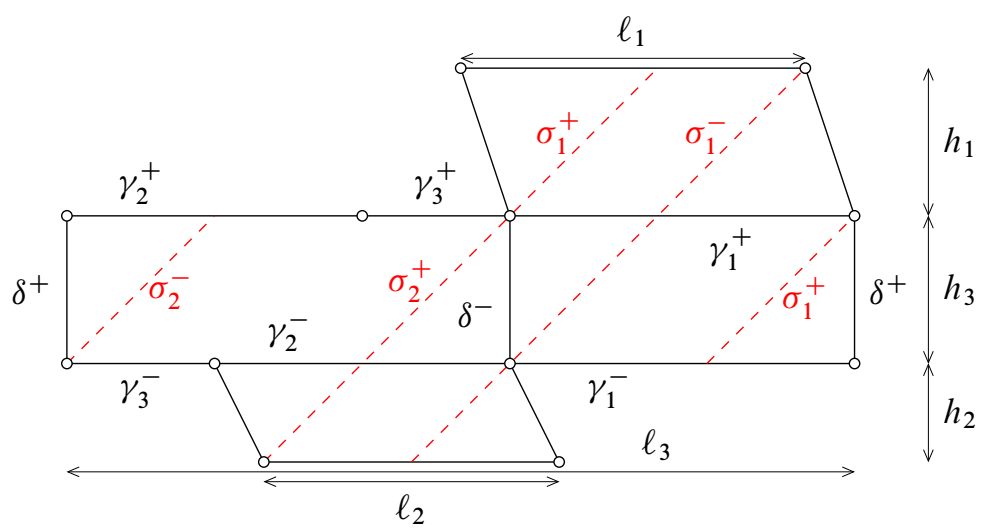

Figure 12: Finding new special splittings

the geodesic segments corresponding to $\sigma^{ \pm}$, we obtain a torus $T$ in $\mathcal{H}(0,0)$ together with a marked saddle connection $\sigma_{2}$. Let $\Lambda$ denote the lattice in $\mathbb{R}^{2}$ associated to $T$. Then $\Lambda$ is generated by $u=\left(\ell_{2}, h_{3}\right)$ and $v=\left(\left|\gamma_{3}^{-}\right|+\left(1-t_{2}\right) \ell_{2},-h_{2}\right)$. Note that $u$ is independent of $t_{2}$.

Recall that $w_{2}$ is parallel to a vector in $\Lambda=\mathbb{Z} u \oplus \mathbb{Z} v$ if and only if we can write $v=\lambda u+\mu w_{2}$ with $\lambda \in \mathbb{Q}$. A direct computation shows that

$$
\lambda=1+\frac{\left|\gamma_{3}^{-}\right|\left(h_{2}+h_{3}\right)}{\ell_{2}\left(h_{2}+h_{3}\right)-t_{2} \ell_{2} h_{3}} .
$$

As $t_{2}$ varies in ]0,1[, we see that the set of $t_{2}$ for which $w_{2}$ is parallel to a vector in $\Lambda$ is countable, which means that, given any $\epsilon>0$, we can find $\left.t_{2} \in\right] 0, \epsilon\left[\right.$ such that $w_{2}$ is not parallel to any vector in $\Lambda$. Therefore, we can find

$$
\left.t_{2} \in\right] 0, \frac{\ell_{1}\left(h_{1}+h_{3}\right)}{\ell_{2}\left(h_{2}+h_{3}\right)}[,
$$

such that $w_{2}$ is not parallel to any vector in $\Lambda$. Now, take

$$
t_{1}=\frac{\ell_{2}\left(h_{2}+h_{3}\right)}{\ell_{1}\left(h_{1}+h_{3}\right)} t_{2}
$$

we have $\left.t_{1} \in\right] 0,1\left[\right.$, hence we can find $\sigma_{1}^{ \pm}$as above. By the choice of $t_{1}$ and $t_{2}$, $w_{1}$ and $w_{2}$ are parallel. Reconstruct $\Sigma$ from $\Sigma^{\prime}$ and $\Sigma^{\prime \prime}$, we see that $\sigma_{1}^{ \pm}$and $\sigma_{2}^{ \pm}$ determine a special splitting of $\Sigma$, which satisfies the condition of Theorem 1.2. Since $\Sigma \in \overline{U \cdot \Sigma_{0}}$, the corollary follows. 


\section{Applications}

\subsection{Generic surfaces with coordinates in a quadratic field}

In [7], Hubert, Lanneau and Möller raise the following question: does there exist a generic translation surface of genus $g$ with all coordinates in a number field $K$ such that $[K: \mathbb{Q}]<g$ ? Theorem 1.2 provides us with an affirmative answer to this question for the case $\mathcal{H}^{\text {hyp }}(4)$. For every quadratic field $K$, one can easily construct a surface in $\mathcal{H}_{1}^{\text {hyp }}(4)$ with all coordinates in $K$ which satisfies the condition of Theorem 1.2. Such a surface with coordinates in $\mathbb{Q}[\sqrt{2}]$ is shown in Figure 13 .

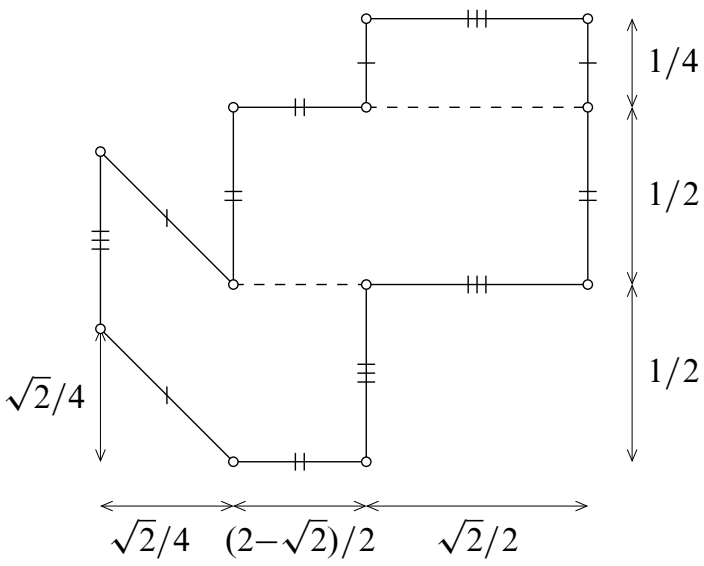

Figure 13: A generic surface in $\mathcal{H}_{1}^{\text {hyp }}(4)$ with coordinates in $\mathbb{Q}[\sqrt{2}]$

\subsection{Thurston-Veech surface with cubic trace field}

Surfaces obtained by the Thurston-Veech construction have large Veech group, which contains infinitely many hyperbolic elements (see [7] for definition and further detail on Thurston-Veech construction). Recall that the trace field of a translation surface is the field generated over $\mathbb{Q}$ by the traces of the matrices in its Veech group. If $K$ is the trace field of a translation surface of genus $g$ then $[K: \mathbb{Q}] \leqslant g$. For $g=2$, McMullen [12] shows that if $[K: \mathbb{Q}]=2$ then the $\operatorname{SL}(2, \mathbb{R})$-orbit of the surface can not be dense in its stratum. However, for $g=3$, Hubert, Lanneau and Möller [6; 5] show that there exist surfaces in the hyperelliptic locus $\mathcal{L}$ of $\mathcal{H}^{\text {odd }}(2,2)$ obtained by the Thurston-Veech construction with trace field of degree 3 such that the $\operatorname{SL}(2, \mathbb{R})$ orbit is dense in $\mathcal{L}$. Note that $\mathcal{L}$ is a closed $\operatorname{SL}(2, \mathbb{R})$-invariant subset of $\mathcal{H}^{\text {odd }}(2,2)$, therefore these surfaces can be viewed as generic. 
The surfaces obtained from Thurston-Veech construction are completely algebraically periodic in the sense of Calta and Smillie [2]. In particular, if such a surface admits a special splitting $\left(T_{1}, T_{2}, T_{3}, v_{1}, v_{2}\right)$, then $v_{2}$ must be parallel to a vector in the lattice associated to $T_{3}$. Therefore, a Thurston-Veech surface can never satisfy the condition of Theorem 1.2. However, if the trace field is of degree 3 over $\mathbb{Q}$, one can find examples of Thurston-Veech surfaces which admit decompositions into three parallel cylinders whose moduli are independent over $\mathbb{Q}$. By Corollary 1.3, it follows that the $\operatorname{SL}(2, \mathbb{R})$-orbits of such surfaces are dense in $\mathcal{H}_{1}^{\text {hyp }}(4)$. Here below, we will give the explicit construction of some of such surfaces.

We construct surfaces in $\mathcal{H}^{\text {hyp }}(4)$ for which the horizontal and vertical directions are completely periodic with three cylinders. To get such a surface, we glue three horizontal cylinders $C_{1}, C_{2}, C_{3}$ as shown in Figure 14.

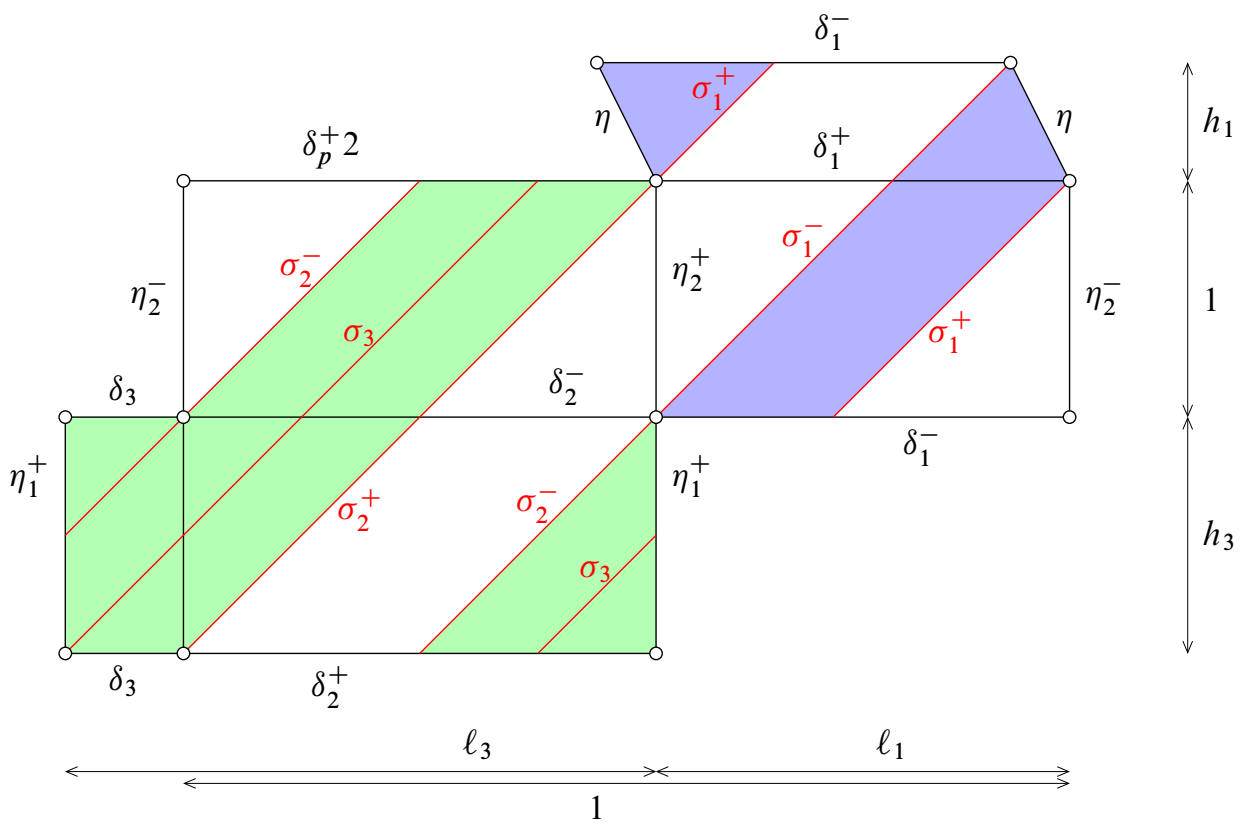

Figure 14: Cubic Thurston-Veech surface with a nonparabolic completely periodic direction

Let $\ell_{i}$ and $h_{i}$ denote the width and the height of $C_{i}, i=1,2,3$. We define the twists $t_{i}$ of $C_{i}, i=1,2,3$, as in Section 8.2.1. In what follows we fix $t_{2}=t_{3}=0$, and consider the cases $t_{1}=(n-1) / n, n \in \mathbb{N}$.

We denote by $\delta_{1}^{ \pm}, \delta_{2}^{ \pm}, \delta_{3}$ the horizontal saddle connections contained in the boundary of $C_{1}, C_{2}, C_{3}$ as shown in Figure 14. We choose the orientation for every horizontal saddle 
connection to be from the left to the right, and for every vertical saddle connection to be upward. Let $\eta_{1}^{-}$(resp. $\eta_{1}^{+}$) denote the vertical saddle connection in $C_{3}$ joining the left (resp. right) endpoints of $\delta_{2}^{+}$and $\delta_{2}^{-}$. Similarly, let $\eta_{2}^{-}$(resp. $\eta_{2}^{+}$) denote the vertical saddle connection in $C_{2}$ joining the left (resp. right) endpoints of $\delta_{2}^{+}$and $\delta_{2}^{-}$. We see that the surface $\Sigma$ admits a special spitting $\left(T_{1}^{\vee}, T_{2}^{\vee}, T_{3}^{\vee}, w_{1}, w_{2}\right)$ determined by $\eta_{1}^{ \pm}$ and $\eta_{2}^{ \pm}\left(w_{i}=V\left(\eta_{i}^{ \pm}\right), i=1,2\right)$. Since $t_{1}=(n-1) / n$, there exists a vertical saddle connection in $T_{3}^{\vee}$ which crosses $\delta_{1}^{-}(n-1)$ times, we denote this saddle connection by $\eta_{3}$. It follows that $\Sigma$ is decomposed into three vertical cylinders, which will be denoted by $C_{i}^{\vee}, i=1,2,3$, where

- $C_{1}^{\vee}$ is bounded by $\eta_{i}^{ \pm}$,

- $C_{2}^{\vee}$ is bounded by $\eta_{1}^{+} \cup \eta_{2}^{+}$and $\eta_{1}^{-} \cup \eta_{2}^{-}$,

- $C_{3}^{\vee}$ is bounded by $\eta_{2}^{+} \cup \eta_{3}$ and $\eta_{2}^{-} \cup \eta_{3}$ ( $\eta_{3}$ bounds $C_{3}^{\vee}$ from both sides).

Fix $\ell_{2}=h_{2}=1$, and let $m_{i}$ denote the modulus of $C_{i}$, and $m_{i}^{\vee}$ denote the modulus of $C_{i}^{\vee}, i=1,2,3$. Set

$$
\begin{aligned}
& a=\frac{m_{1}}{m_{2}}=\frac{h_{1}}{\ell_{1}}, \\
& b=\frac{m_{3}}{m_{2}}=\frac{h_{3}}{\ell_{3}}, \\
& c=\frac{m_{2}^{\vee}}{m_{1}^{\vee}}=\frac{h_{3}\left(1-\ell_{1}\right)}{\left(h_{3}+1\right)\left(\ell_{1}+\ell_{3}-1\right)}, \\
& d=\frac{m_{2}^{\vee}}{m_{3}^{\vee}}=\frac{n^{2}\left(h_{1}+1\right)\left(1-\ell_{1}\right)}{\left(h_{3}+1\right) \ell_{1}} .
\end{aligned}
$$

Let $\eta$ denote the saddle connection in $C_{1}$ such that $V(\eta)=\left(-\ell_{1} / n, h_{1}\right)$. We have a pair of homologous saddle connections $\sigma_{1}^{ \pm}$in $T_{3}^{\vee}$ such that

$$
\sigma_{1}^{+}=\sigma_{1}^{-}=\delta_{1}^{-}+\eta_{2}^{-}+\eta
$$

in $H_{1}(\Sigma, \mathbb{Z})$. Note that $\sigma_{1}^{ \pm}$bound a cylinder containing $\eta$. Similarly, we have in $T_{2}^{\vee}$ a pair of homologous saddle connections $\sigma_{2}^{ \pm}$such that

We have

$$
\sigma_{2}^{+}=\sigma_{2}^{-}=\delta_{2}^{+}+\eta_{1}^{+}+\eta_{2}^{+} \text {. }
$$

$$
V\left(\sigma_{1}^{ \pm}\right)=\left(\frac{n-1}{n} \ell_{1}, h_{1}+1\right), V\left(\sigma_{2}^{ \pm}\right)=\left(1-\ell_{1}, h_{3}+1\right)
$$

Claim $1 V\left(\sigma_{1}^{+}\right)$and $V\left(\sigma_{2}^{+}\right)$are colinear if and only if $d=n(n-1)$. 
Proof The fact that $V\left(\sigma_{1}^{+}\right)$is parallel to $V\left(\sigma_{2}^{+}\right)$is equivalent to

$$
\begin{array}{rlrl}
\frac{n-1}{n} \frac{\ell_{1}}{1-\ell_{1}} & =\frac{1+h_{1}}{1+h_{3}} \\
& \Longleftrightarrow \quad \frac{1-\ell_{1}}{1+h_{3}} \frac{1+h_{1}}{\ell_{1}} & =\frac{n-1}{n} \\
& \Longleftrightarrow \frac{d}{n^{2}} & =\frac{n-1}{n} \\
d & \Longleftrightarrow n(n-1) .
\end{array}
$$

Clearly, the surface $\Sigma$ is completely determined by the values of $\left(h_{1}, \ell_{1}, h_{3}, \ell_{3}\right)$. We will find some values of $\left(h_{1}, \ell_{1}, h_{3}, \ell_{3}\right)$ such that the vertical and horizontal directions are parabolic, ie $a, b, c, d$ are rational numbers, and $\Sigma$ admits a decomposition into three cylinders in the direction $V\left(\sigma_{1}^{ \pm}\right)$whose moduli are independent over $\mathbb{Q}$. For this purpose, we fix $n \in \mathbb{N}, a, b, c$ in $\mathbb{Q}$, and $d=n(n-1)$, then we compute $\left(h_{1}, \ell_{1}, h_{3}, \ell_{3}\right)$ as functions of $(n, a, b, c)$.

First, observe that since $c=m_{2}^{\vee} / m_{1}^{\vee}$ is a rational number, the vector $V\left(\sigma_{2}^{+}\right)$is parallel to a vector in the lattice associated to $T_{1}^{\vee}$. It follows that $\Sigma$ is decomposed into three cylinders in the direction $V\left(\sigma_{1}^{+}\right)$. We denote these cylinders by $C_{i}^{\prime}, i=1,2,3$, where

- $C_{1}^{\prime}$ is the cylinder containing $\eta$, and bounded by $\sigma_{1}^{ \pm}$,

- $C_{2}^{\prime}$ is the cylinder containing $\eta_{2}^{ \pm}$, and bounded by $\sigma_{1}^{+} \cup \sigma_{2}^{+}$and $\sigma_{1}^{-} \cup \sigma_{2}^{-}$,

- $C_{3}^{\prime}$ is the complement of $C_{1}^{\prime} \cup C_{2}^{\prime}$, which is bounded by $\sigma_{2}^{+} \cup \sigma_{3}$ and $\sigma_{2}^{-} \cup \sigma_{3}$, where $\sigma_{3}$ is a saddle connection parallel to $\sigma_{2}^{ \pm}$, and bounds $C_{3}^{\prime}$ from both sides.

Let $m_{i}^{\prime}, i=1,2,3$, denote the modulus of $C_{i}^{\prime}$.

Claim 2 Suppose that $c \in \mathbb{N}$. Then we have

$$
\begin{aligned}
& \frac{m_{1}^{\prime}}{m_{2}^{\prime}}=\frac{1}{(n-1)^{2}}\left(n a \ell_{1}+1\right)\left(\frac{n}{\ell_{1}}-1\right), \\
& \frac{m_{3}^{\prime}}{m_{2}^{\prime}}=\frac{b}{n c^{2}}\left(n-\ell_{1}\right) .
\end{aligned}
$$

Proof Let $h_{i}^{\prime}, \ell_{i}^{\prime}, i=1,2,3$, denote the height and the width of $C_{i}^{\prime}$. We have

$$
\frac{h_{1}^{\prime}}{h_{2}^{\prime}}=\frac{\operatorname{Area}\left(C_{1}^{\prime}\right)}{\operatorname{Area}\left(T_{3}^{\vee}\right)-\operatorname{Area}\left(C_{1}^{\prime}\right)}=\frac{\left(h_{1}+1\right) \ell_{1}-((n-1) / n) \ell_{1}}{((n-1) / n) \ell_{1}}=\frac{1+n h_{1}}{n-1} .
$$


Using the vertical projection onto the horizontal axis, we have

$$
\frac{\ell_{2}^{\prime}}{\ell_{1}^{\prime}}=1+\frac{1-\ell_{1}}{((n-1) / n) \ell_{1}}=\frac{n-\ell_{1}}{(n-1) \ell_{1}} .
$$

Therefore

$$
\frac{m_{1}^{\prime}}{m_{2}^{\prime}}=\frac{h_{1}^{\prime}}{h_{2}^{\prime}} \frac{\ell_{2}^{\prime}}{\ell_{1}^{\prime}}=\frac{1+n h_{1}}{(n-1)} \frac{n-\ell_{1}}{(n-1) \ell_{1}}=\frac{1}{(n-1)^{2}}\left(1+n a \ell_{1}\right) \frac{n-\ell_{1}}{\ell_{1}} .
$$

Rescaling so that $V\left(\delta_{3}\right)=(1,0)$ and $V\left(\eta_{1}\right)=(0,1)$, that is $m_{1}^{\vee}=1$. Since $m_{2}^{\vee} / m_{1}^{\vee}=c$, we deduce that $V\left(\sigma_{2}^{+}\right)$is collinear with the vector $(c, 1)$. When $c$ is an integer, in the standard torus $\mathbb{R}^{2} / \mathbb{Z}^{2}$, the number of intersection points of the simple closed geodesics corresponding to the vectors $(c, 1)$ and $(0,1)$ is given by $|(c, 1) \wedge(0,1)|=c$. Therefore, the saddle connection $\sigma_{3}$ crosses $\eta_{1}^{+} c$ times. Using the projection along $V\left(\sigma_{1}^{ \pm}\right)$onto the vertical axis we have $h_{3}^{\prime} / h_{2}^{\prime}=h_{3} / c$. Then using the vertical projection onto the horizontal axis, we have $\ell_{2}^{\prime} / \ell_{3}^{\prime}=\left(1-\ell_{1} / n\right) /\left(c \ell_{3}\right)$. Therefore,

$$
\frac{m_{3}^{\prime}}{m_{2}^{\prime}}=\frac{h_{3}^{\prime}}{h_{2}^{\prime}} \frac{\ell_{2}^{\prime}}{\ell_{3}^{\prime}}=\frac{1}{n c^{2}} \frac{h_{3}}{\ell_{3}}\left(n-\ell_{1}\right)=\frac{b}{n c^{2}}\left(n-\ell_{1}\right) .
$$

Given $n \in \mathbb{N}, n>1, a, b \in \mathbb{Q}, a>0, b>0$, and $c \in \mathbb{N}, c>0$, set

$$
\begin{aligned}
& P(X)=\frac{n c}{n-1}(X+a)\left(\frac{n}{(n-1) b}(X-1)(X+a)-\frac{X}{b}-X+1\right) \\
&-\frac{n}{n-1}(X-1)(X+a)+X .
\end{aligned}
$$

Claim 3 Suppose that $P(X)$ is irreducible over $\mathbb{Q}$, and has a real root $\alpha$ satisfying

$$
\begin{gathered}
\alpha>1, \\
\frac{n(\alpha-1)(\alpha+a)}{(n-1) \alpha}>1 .
\end{gathered}
$$

Then by taking

$\ell_{1}=\frac{1}{\alpha}, \quad h_{1}=\frac{a}{\alpha}, \quad \ell_{3}=\frac{1}{b}\left(\frac{n(\alpha-1)(\alpha+a)}{(n-1) \alpha}-1\right), \quad h_{3}=\frac{n(\alpha-1)(\alpha+a)}{(n-1) \alpha}-1$,

the construction above gives us a Thurston-Veech surface with trace field of degree 3 over $\mathbb{Q}$ for which the moduli of the three cylinders in the direction $V\left(\sigma_{1}^{ \pm}\right)$are independent over $\mathbb{Q}$. Consequently, this surface is generic in $\mathcal{H}^{\text {hyp }}(4)$ by Corollary 1.3. 
Proof From the choice of $\ell_{1}, h_{1}, \ell_{3}, h_{3}$, we only need to check that

$$
\begin{aligned}
& \ell_{1}+\ell_{3}-1>0, \\
& \frac{m_{2}^{\vee}}{m_{1}^{\vee}}=\frac{1-\ell_{1}}{1+h_{3}} \frac{h_{3}}{\ell_{1}+\ell_{3}-1}=c, \\
& \frac{m_{2}^{\vee}}{m_{3}^{\vee}}=\frac{n^{2}\left(1-\ell_{1}\right)\left(1+h_{1}\right)}{\ell_{1}\left(1+h_{3}\right)}=n(n-1) .
\end{aligned}
$$

Condition (a) is satisfied since we have

$$
\begin{aligned}
\ell_{1}+\ell_{3}-1 & =\frac{1}{\alpha}+\frac{n}{b(n-1)} \frac{(\alpha-1)(\alpha+a)}{\alpha}-\frac{1}{b}-1 \\
& =\frac{n-1}{c n(\alpha+a)}\left(\frac{n}{n-1} \frac{(\alpha-1)(\alpha+a)}{\alpha}-1\right)>0 .
\end{aligned}
$$

Conditions (b) and (c) follow immediately from the fact that $\alpha$ is a root of $P$. To see that the trace field of $\Sigma$ is of degree 3 , remark that we have $m_{i} \in \mathbb{Q}, i=1,2,3$, but

$$
m_{3}^{\vee}=\frac{\ell_{1}}{n^{2}\left(1+h_{1}\right)}=\frac{1}{n^{2}(\alpha+a)} .
$$

is an algebraic number of degree 3 over $\mathbb{Q}$. From Claim 2, we have

$$
\begin{aligned}
\frac{m_{1}^{\prime}}{m_{2}^{\prime}} & =\frac{1}{(n-1)^{2}}\left(1+n a \ell_{1}\right)\left(\frac{n}{\ell_{1}}-1\right) \\
& =\frac{1}{(n-1)^{2}}\left(1+\frac{n a}{\alpha}\right)(n \alpha-1) \\
& =\frac{n}{(n-1)^{2}} \alpha+\frac{n^{2} a-1}{(n-1)^{2}}-\frac{n a}{(n-1)^{2}} \frac{1}{\alpha} .
\end{aligned}
$$

and

$$
\begin{aligned}
\frac{m_{3}^{\prime}}{m_{2}^{\prime}} & =\frac{b}{n c^{2}}\left(n-\ell_{1}\right) \\
& =\frac{b}{c^{2}}-\frac{b}{n c^{2}} \frac{1}{\alpha} .
\end{aligned}
$$

Since $\alpha, 1,1 / \alpha$ are independent over $\mathbb{Q}$, it follows that $m_{1}^{\prime}, m_{2}^{\prime}, m_{3}^{\prime}$ are independent over $\mathbb{Q}$.

Below are some explicit examples of Thurston-Veech surfaces obtained from the construction above which satisfy the condition of Corollary 1.3 . Here $\widetilde{P}(X)$ is a polynomial proportional to $P(X)$ with coefficients in $\mathbb{Z}$ and values of $\alpha, \ell_{1}$ and $\ell_{3}$ are approximate. 


\begin{tabular}{|c|c|c|c|c|}
\hline$(n, a, b, c)$ & $\widetilde{P}(X)$ & $\alpha$ & $\ell_{1}$ & $\ell_{3}$ \\
\hline$(4,1,10,5)$ & $8 X^{3}-70 X^{2}-5 X+64$ & 8.716407 & 0.114726 & 1.046891 \\
$(5,2,10,3)$ & $15 X^{3}-127 X^{2}-152 X+260$ & 9.352026 & 0.106929 & 1.167271 \\
$(5,1 / 5,2,1)$ & $25 X^{3}-115 X^{2}+83 X+15$ & 3.643625 & 0.274452 & 1.242959 \\
$(5,1 / 2,5,1)$ & $20 X^{3}-176 X^{2}+121 X+75$ & 7.983332 & 0.125261 & 1.655175 \\
$(2,1,6,1)$ & $2 X^{3}-11 X^{2}+10$ & 5.323574 & 0.187844 & 1.545243 \\
$(2,2,9,2)$ & $8 X^{3}-34 X^{2}-53 X+76$ & 5.175414 & 0.193221 & 1.175327 \\
\hline
\end{tabular}

\section{References}

[1] K Calta, Veech surfaces and complete periodicity in genus two, J. Amer. Math. Soc. 17 (2004) 871-908 MR2083470

[2] K Calta, J Smillie, Algebraically periodic translation surfaces, J. Mod. Dyn. 2 (2008) 209-248 MR2383267

[3] A Douady, J Hubbard, On the density of Strebel differentials, Invent. Math. 30 (1975) 175-179 MR0396936

[4] A Eskin, A Okounkov, Asymptotics of numbers of branched coverings of a torus and volumes of moduli spaces of holomorphic differentials, Invent. Math. 145 (2001) 59-103 MR1839286

[5] P Hubert, E Lanneau, M Möller, Completely periodic directions and orbit closures of many pseudo-Anosov Teichmïler discs in $Q(1,1,1,1)$, to appear in Math. Ann. arXiv : math.GT/0611655

[6] P Hubert, E Lanneau, M Möller, The Arnoux-Yoccoz Teichmüller disc, Geom. Funct. Anal. 18 (2009) 1988-2016 MR2491696

[7] P Hubert, E Lanneau, M Möller, $\mathrm{GL}_{2}^{+}(\mathbb{R})$-orbit closures via topological splittings, from: "Surveys in differential geometry, Vol. XIV: Geometry of Riemann surfaces and their moduli spaces", (L Ji, S A Wolpert, S-T Yau, editors), Surv. Differ. Geom. 14, Int. Press, Somerville, MA (2009) 145-169 MR2655326

[8] P Hubert, T A Schmidt, An introduction to Veech surfaces, from: "Handbook of dynamical systems. Vol. 1B", (B Hasselblatt, A Katok, editors), Elsevier, Amsterdam (2006) 501-526 MR2186246

[9] M Kontsevich, A Zorich, Connected components of the moduli spaces of Abelian differentials with prescribed singularities, Invent. Math. 153 (2003) 631-678 MR2000471

[10] H Masur, Interval exchange transformations and measured foliations, Ann. of Math. (2) 115 (1982) 169-200 MR644018

[11] H Masur, S Tabachnikov, Rational billiards and flat structures, from: "Handbook of dynamical systems, Vol. 1A”, (B Hasselblatt, A Katok, editors), North-Holland, Amsterdam (2002) 1015-1089 MR1928530 
[12] C T McMullen, Dynamics of $\mathrm{SL}_{2}(\mathbb{R})$ over moduli space in genus two, Ann. of Math. (2) 165 (2007) 397-456 MR2299738

[13] W P Thurston, On the geometry and dynamics of diffeomorphisms of surfaces, Bull. Amer. Math. Soc. (N.S.) 19 (1988) 417-431 MR956596

[14] W A Veech, Teichmüller curves in moduli space, Eisenstein series and an application to triangular billiards, Invent. Math. 97 (1989) 553-583 MR1005006

[15] W A Veech, Moduli spaces of quadratic differentials, J. Analyse Math. 55 (1990) 117-171 MR1094714

[16] A Zorich, Flat surfaces, from: "Frontiers in number theory, physics, and geometry I", (P Cartier, B Julia, P Moussa, P Vanhove, editors), Springer, Berlin (2006) 437-583 MR2261104

Institut de Mathématiques de Bordeaux, Bat A33, Université Bordeaux 1

351, cours de la Libération, F-33405 Talence Cedex, France

duc-manh.nguyen@math.u-bordeaux1.fr

http://www . math.u-bordeaux1.fr/ nguyendm/

Proposed: Benson Farb

Seconded: Walter Neumann, Joan Birman
Received: 6 December 2010

Revised: 12 September 2011 